\title{
Multi-line (sub)millimetre observations of the high-mass proto cluster IRAS 05358+3543*
}

\author{
S. Leurini ${ }^{1,2}$, H. Beuther ${ }^{3}$, P. Schilke ${ }^{1}$, F. Wyrowski ${ }^{1}$, Q. Zhang ${ }^{4}$, and K. M. Menten ${ }^{1}$ \\ 1 Max-Planck-Institut für Radioastronomie, Auf dem Hügel 69, 53121 Bonn, Germany \\ 2 ESO, Karl-Schwarzschild Strasse 2, 85748 Garching-bei-München, Germany \\ e-mail: sleurini@eso.org \\ 3 Max-Planck-Institut für Astronomie, Königstuhl 17, 69117 Heidelberg, Germany \\ 4 Harvard-Smithsonian Center for Astrophysics, 60 Garden Street, Cambridge, MA 02138, USA
}

Received 30 May 2007 / Accepted 20 September 2007

\section{ABSTRACT}

\begin{abstract}
Context. Since most high- and intermediate-mass protostars are at great distance and form in clusters, high linear resolution observations are needed to investigate their physical properties.

Aims. To study the gas in the innermost region around the protostars in the proto-cluster IRAS 05358+3543, we observed the source in several transitions of methanol and other molecular species with the Plateau de Bure Interferometer and the Submillimeter Array, reaching a linear resolution of $1100 \mathrm{AU}$.

Methods. We determine the kinetic temperature of the gas around the protostars through an LVG and LTE analysis of their molecular emission; the column densities of $\mathrm{CH}_{3} \mathrm{OH}, \mathrm{CH}_{3} \mathrm{CN}$ and $\mathrm{SO}_{2}$ are also derived. Constraints on the density of the gas are estimated for two of the protostellar cores.

Results. We find that the dust condensations are in various evolutionary stages. The powerhouse of the cluster, mm1a, harbours a hot core with $T \sim 220(75<T<330) \mathrm{K}$. A double-peaked profile is detected in several transitions toward mm 1 a, and we found a velocity gradient along a linear structure which could be perpendicular to one of the outflows from the vicinity of mm1a. Since the size of the double-peaked emission is less than $1100 \mathrm{AU}$, we suggest that mm1a might host a massive circumstellar disk. The other sources are in earlier stages of star formation. The least active source, $\mathrm{mm} 3$, could be a starless massive core, since it is cold $(T<20 \mathrm{~K})$, with a large reservoir of accreting material $\left(M \sim 19 M_{\odot}\right)$, but no molecular emission peaks on it.
\end{abstract}

Key words. stars: formation - stars: early type - stars: individual: IRAS 05358+3543 - ISM: molecules

\section{Introduction}

The last decade has seen significant progress in the understanding of how high-mass stars form. Large samples of massive young stellar objects (YSOs) were studied with single-dish telescopes, to investigate their physical properties through the analysis of their (sub)mm continuum and molecular emission (e.g., Molinari et al. 1996, 1998, 2000; Walsh et al. 1997, 1998, 1999; Hatchell et al. 2000; Zhang et al. 2001, 2005; Sridharan et al. 2002; Beuther et al. 2002b,c; Faúndez et al. 2004; Williams et al. 2004, 2005). However, an intrinsic feature of high-mass stars is that they form in clusters, and that most of them are at large (several kpc) distances. Therefore, single-dish studies, as valuable as they are, lack the necessary spatial resolution to resolve single protostars and study the inner regions where high-mass star formation takes place. Interferometric observations started shedding light into the complex nature of high-mass star forming regions with the adequate spatial resolution (e.g., Cesaroni et al. 1997, 1999, 2005; Wyrowski et al. 1999; Beuther et al. 2002a, 2005; Hunter et al. 2006; Shepherd et al. 2000). However, the number of massive YSOs studied at high resolution is still too small to establish the general properties of the dense cores where massive stars form on a statistical base.

* UV tables of interferometer maps plus IRAM 30-m map are only available in electronic form at the CDS via anonymous ftp to cdsarc.u-strasbg.fr $(130.79 .128 .5)$ or via http://cdsweb.u-strasbg.fr/cgi-bin/qcat? J/A+A/475/925
In this paper, we present an interferometric analysis of the high-mass star forming region IRAS $05358+3543$ at (sub)mm wavelengths in several molecular transitions. IRAS 05358+3543 (also known in literature as S233IR) is part of a sample of 69 high-mass protostellar objects studied in great detail in recent years (Sridharan et al. 2002; Beuther et al. 2002b,c,d; Williams et al. 2004, 2005; Fuller et al. 2005). At a distance of $1.8 \mathrm{kpc}$ (Snell et al. 1990), IRAS $05358+3543$ has a bolometric luminosity of $6300 L_{\odot}$; strong high-mass star formation activity is evidenced by maser emission (see Menten 1991; Tofani et al. 1995; Minier et al. 2000) and outflow activity (Snell et al. 1990; Beuther et al. 2002a). Previous interferometric observations by Beuther et al. (2002a) resolved three dust condensations (mm1, $\mathrm{mm} 2$ and mm3) within an area of $9^{\prime \prime} \times 4^{\prime \prime}(\sim 17100 \times 7200 \mathrm{AU})$, and revealed at least three outflows in $\mathrm{CO}$ and $\mathrm{SiO}$, the most prominent of which is more than a parsec in length, and massive $\left(M>10 M_{\odot}\right)$. Two of the three identified outflows originate from the vicinity of $\mathrm{mm} 1$, which is probably the main powerhouse in the region.

To zoom in on the innermost region around the protostars, and study the physical properties of the individual potentially star-forming cores, we carried out a comprehensive program to observe the region at high spatial resolution with the Plateau de Bure Interferometer ${ }^{1}$ at $97 \mathrm{GHz}$ and $241 \mathrm{GHz}$, and the

1 IRAM is supported by INSU/CNRS (France), MPG (Germany) and IGN (Spain). 
Table 1. Positions of the four dust condensations in IRAS $05358+3543$ (from Beuther et al. 2007a).

\begin{tabular}{lcc}
\hline \hline Source & RA [J2000] & Dec [J2000] \\
\hline mm1a & $05: 39: 13.08$ & $35: 45: 51.3$ \\
mm1b & $05: 39: 13.13$ & $35: 45: 50.8$ \\
mm2a & $05: 39: 12.76$ & $35: 45: 51.3$ \\
mm3 & $05: 39: 12.50$ & $35: 45: 54.9$ \\
\hline
\end{tabular}

Submillimeter Array ${ }^{2}$ at $338 \mathrm{GHz}$. The new observations reach a resolution down to $0.6^{\prime \prime}$, corresponding to $\sim 1100 \mathrm{AU}$ at the distance of the source. Beuther et al. (2007a) studied the continuum emission of this dataset. They identified four compact protostellar sources in the region; $\mathrm{mm} 1$ is resolved into two continuum peaks, mm $1 \mathrm{a}$ and $\mathrm{mm} 1 \mathrm{~b}$, with a projected linear separation of 1700 AU. A mid-infrared source (Longmore et al. 2006), and a compact $3.6 \mathrm{~cm}$ continuum source (Beuther et al. 2007a) coincide with mm1a, which is also associated with the class II methanol masers detected by Minier et al. (2000). The previously identified source $\mathrm{mm} 2$ resolves into several sub-sources; however, only one of them ( $\mathrm{mm} 2 \mathrm{a}$, according to the nomenclature used by Beuther et al. 2007a) is a protostellar source, while the others are probably caused by the outflows in the region. The third source $\mathrm{mm} 3$ remains a single compact core even at the highest spatial resolution. In Table 1, we report the positions of the four sources identified in the continuum emission by Beuther et al. (2007a).

In this paper, we discuss the spectral line observations complementing the continuum data discussed by Beuther et al. (2007a). In Sect. 2, the different observations are presented. In Sect. 3, we discuss our results, and analyse the extended emission of low excitation molecular transitions (Sect. 3.1), as well as the molecular spectra at the positions of the dust condensations (Sect. 3.2). Finally, in Sect. 4 we derive the physical parameters of the gas around the protostars from the analysis of their spectra. In the following sections, we use the term protostar for young massive stellar objects which are still accreting material from the surroundings, independently of whether they already started burning hydrogen or not.

\section{Observations}

\subsection{Plateau de Bure Interferometer (PdBI)}

IRAS $05358+3543$ was observed with the IRAM Plateau de Bure Interferometer in two different frequency setups, in 2003 and 2005. A first frequency setup was performed in four tracks between January and October 2003 in the BC and D configurations of the array. Two observations, in January and October, were performed with only 5 antennas; on January 12th, 2003, the array was configured in a special $\mathrm{BC}$ combination. The $3 \mathrm{~mm}$ receivers were used in single-sided band mode and tuned to $96.6 \mathrm{GHz}$; the $1 \mathrm{~mm}$ receivers, in double-sided band mode, were tuned to $241.85 \mathrm{GHz}$ (USB). At $3 \mathrm{~mm}$, the $\mathrm{C}^{34} \mathrm{~S}$ line and the torsionally excited $2_{k} \rightarrow 1_{k} \mathrm{CH}_{3} \mathrm{OH}$ quartet were covered using two correlator units of $80 \mathrm{MHz}$ bandwidth. One $320 \mathrm{MHz}$ unit was placed to obtain a continuum measurement at $3 \mathrm{~mm}$. The $5_{k} \rightarrow 4_{k} v_{t}=0 \mathrm{CH}_{3} \mathrm{OH}$ band, the $2_{1,1} \rightarrow 2_{1,2} \mathrm{HDO}$ and the $5 \rightarrow 4 \mathrm{SO}_{2}$ lines were observed with four units of $160 \mathrm{MHz}$

2 The Submillimeter Array is a joint project between the Smithsonian Astrophysical Observatory and the Academia Sinica Institute of Astronomy and Astrophysics and is funded by the Smithsonian Institution and the Academia Sinica. bandwidth, which were also used to obtain a continuum measurement at $1.3 \mathrm{~mm}$. The same configuration of the correlator units allowed the observation of $13_{k} \rightarrow 12_{k} \mathrm{CH}_{3} \mathrm{CN}$ band in the LSB at $239 \mathrm{GHz}$. The observations were performed in the MOSAIC mode, with seven fields covering the whole source in a hexagonal pattern with a centre field (see Fig. 1).

The second frequency setup was observed on February 4th and February 7th, 2005, in the A and B configurations. The $3 \mathrm{~mm}$ receivers were used in single-side band mode and tuned again to $96.6 \mathrm{GHz}$, with the same configuration of the correlator described before. The $1 \mathrm{~mm}$ receivers, in double side-band mode, were tuned to $241.2 \mathrm{GHz}$ (USB) with three $160 \mathrm{MHz}$ units covering the $5_{k} \rightarrow 4_{k} v_{t}=1 \mathrm{CH}_{3} \mathrm{OH}$ band.

Bandpass calibration was done with 0420-014, 3C 454.2 and NRAO150 for the BCD configuration, with 3C 84 for the AB data. NRAO150, 0528+134 and 3C 273 were used as flux calibrators of the $\mathrm{BCD}$ and $\mathrm{AB}$ data, respectively. Phase and amplitude calibration was done via observations of $0528+134$, $0552+398,0529+483$ and J0418+380. Measured system temperatures were between 100 and $260 \mathrm{~K}$ in the $3.1 \mathrm{~mm}$ band for both setups. On February 4th, the system temperatures in the $1.2 \mathrm{~mm}$ receivers ranged between 240 and $420 \mathrm{~K}$, with one receiver measuring system temperatures of $900 \mathrm{~K}$. On February 7 th, and during the observations of the first frequency setup, the system temperatures in the $1.2 \mathrm{~mm}$ bands were higher, between 400 and $1000 \mathrm{~K}$, due to less favourable weather conditions.

For both frequency setups, the phase centre was $\alpha_{2000}=$ $05^{\mathrm{h}} 39^{\mathrm{m}} 13.07, \delta_{2000}=+35^{\circ} 45^{\prime} 50^{\prime} .5$, with $v_{\mathrm{LSR}}=-17.6 \mathrm{~km} \mathrm{~s}^{-1}$. Details on the spectral resolutions, the synthesised beam sizes and the main spectral lines per frequency units are given in Table 2. For the $\mathrm{AB}$ configuration the baselines range between 30 and $400 \mathrm{~m}$, for the BCD configuration between 20 and $320 \mathrm{~m}$. Therefore, at $1.2 \mathrm{~mm}$ any source structure larger than $6^{\prime \prime}$ is filtered out in the $\mathrm{AB}$ observations, and larger than $13^{\prime \prime}$ in the BCD data.

The data calibration and the imaging were performed with the CLIC and MAPPING software ${ }^{3}$. For the PdBI and the SMA data, line free channels were averaged to produce continuum images, which were then subtracted from the line data in the visibility plane ${ }^{4}$.

\subsection{Submillimeter Array}

We observed IRAS $05358+3543$ with the SMA on November 11th, 2004, at $348 \mathrm{GHz}(865 \mu \mathrm{m})$ in the compact configuration with seven antennas, and on January 15 th, 2005 , in the extended configuration again with seven antennas in the array. However, the data from one antenna in the extended configuration were unusable reducing it to six effective antennas for that configuration. Due to problems at the correlator during the observations in the extended configuration, the frequency of the $7 \rightarrow 6 \mathrm{C}^{34} \mathrm{~S}$ line was not covered. However, the $7 \rightarrow$ $6 \mathrm{C}^{34} \mathrm{~S}$ map was produced from the compact configuration data. The projected baselines ranged between 13 and $223 \mathrm{~m}$. The short baseline cutoff implies that source structures $\geq 16^{\prime \prime}$ are filtered out by the observations. The phase centre of the observations was $\alpha_{2000}=05^{\mathrm{h}} 39^{\mathrm{m}} 13^{\mathrm{s}} .07$ and $\delta_{2000}=+35^{\circ} 45^{\prime} 51^{\prime \prime} .2$

\footnotetext{
${ }^{3}$ http://wWw.iram.fr/IRAMFR/GILDAS

4 The calibrated UV tables related to the PdBI and SMA observations are available in electronic form at the CDS. The integrated intensity map of the $5_{0} \rightarrow 4_{0} v_{t}=0 \mathrm{CH}_{3} \mathrm{OH}-A$ line with the $30 \mathrm{~m}$ IRAM is also available in electronic form at the CDS.
} 


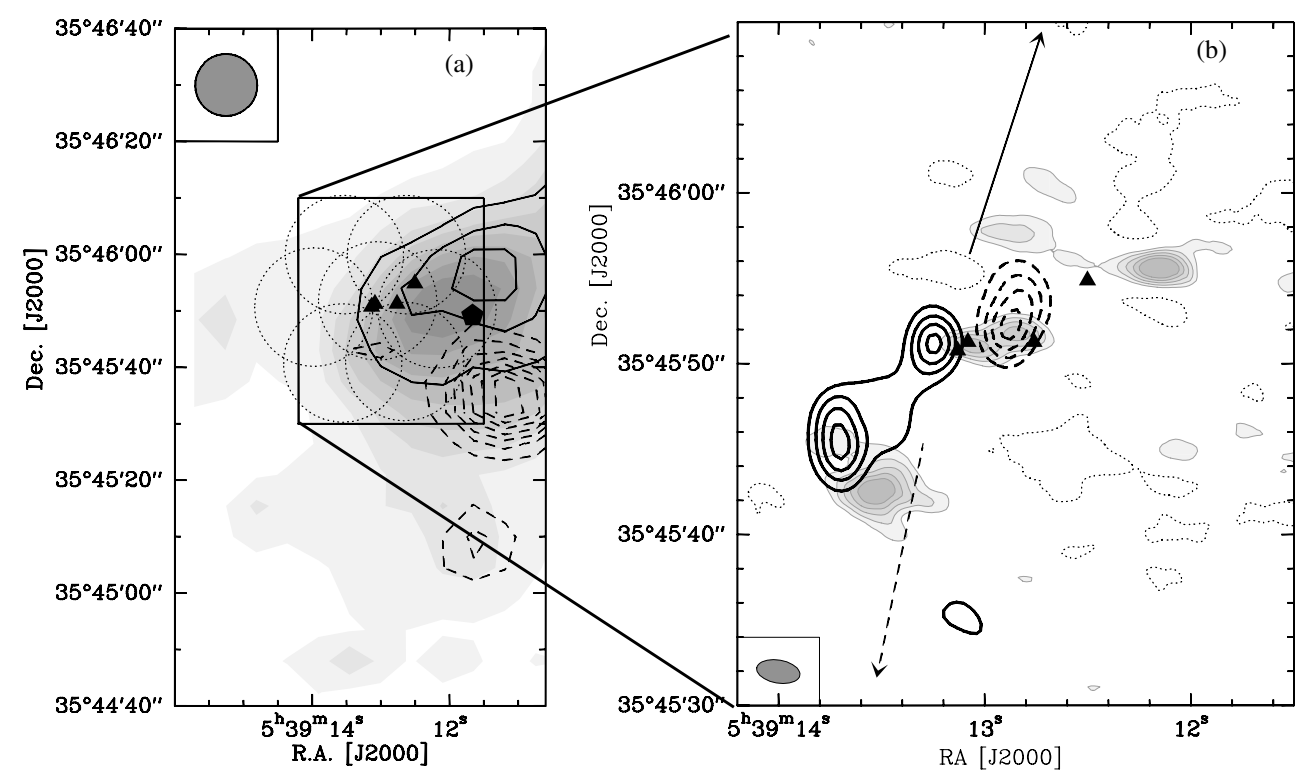

Fig. 1. a) In grey scale, the map of the integrated intensity of the $5_{0} \rightarrow 4_{0} v_{t}=0 \mathrm{CH}_{3} \mathrm{OH}-A$ line with the $30 \mathrm{~m}$ IRAM telescope $(v=$ $[-17,-16] \mathrm{km} \mathrm{s}^{-1}$; levels from $0.5 \mathrm{Jy}_{\text {beam }}^{-1} \mathrm{~km} \mathrm{~s}^{-}$in steps of 0.5$)$. The dashed contours show the blue-shifted emission $\left(v=[-23,-20] \mathrm{km} \mathrm{s}^{-1}\right.$; levels from $0.5 \mathrm{Jy} \mathrm{beam}^{-1} \mathrm{~km} \mathrm{~s}^{-1}$ in steps of 0.5$)$; the solid lines the red-shifted emission $\left(v=[-14,-12] \mathrm{km} \mathrm{s}^{-1}\right.$; levels from $1 \mathrm{Jy}$ beam ${ }^{-1} \mathrm{~km} \mathrm{~s}^{-1}$ in steps of 0.5 ). The beam is indicated in the top left corner. The dotted circles outline the observed mosaic of seven fields. The pentagon locates the position of one of the $\mathrm{H}^{13} \mathrm{CO}^{+}$peaks observed by Beuther et al. (2002a), which is the candidate centre for the outflow traced by $\mathrm{CH}_{3} \mathrm{OH}$. The solid lines outline the region shown in panel b). b) In grey scale (and grey contours), the map of the integrated intensity of the $5_{0} \rightarrow 4_{0} v_{t}=0 \mathrm{CH} \mathrm{H}_{3} \mathrm{OH}-\mathrm{A}$ line with the Plateau de Bure Interferometer $\left(v=[-24,-12] \mathrm{km} \mathrm{s}^{-1}\right)$. Level contours are from $1 \mathrm{Jy} \mathrm{beam}^{-1} \mathrm{~km} \mathrm{~s}^{-1}$, in step of 0.5 . Dotted contours show the negative emission $\left(-0.8 \mathrm{Jy} \mathrm{beam}^{-1} \mathrm{~km} \mathrm{~s}^{-1}\right.$ ). The black contours outline the high velocity outflow (CO $2 \rightarrow 1$ SMA data, Beuther priv. comm. Blue-shifted emission $v=[-44,-24] \mathrm{km} \mathrm{s}^{-1}$; red-shifted emission $v=[-8,2] \mathrm{km} \mathrm{s}^{-1}$; levels from 30 to $100 \mathrm{Jy} \mathrm{beam}^{-1} \mathrm{~km} \mathrm{~s}^{-1}$ in steps of 20). The four triangles mark the positions of the main $\mathrm{mm}$ dust condensations. For illustration, the direction of the highly collimated outflow (Beuther et al. 2002a) is shown by the arrows. For both flows, dashed lines are for the blue-shifted emission, solid lines for the red-shifted emission. The beam is indicated in the bottom left corner.

Table 2. Observational parameters.

\begin{tabular}{lrccccr}
\hline \hline \multicolumn{1}{c}{ Main line } & $\begin{array}{c}\text { Centre frequency } \\
(\mathrm{GHz})\end{array}$ & Configuration & $\begin{array}{c}\text { HPBW } \\
\left({ }^{\prime \prime}\right)\end{array}$ & $\begin{array}{c}\text { PA } \\
\left({ }^{\circ}\right)\end{array}$ & $\begin{array}{c}\Delta v \\
\left(\mathrm{~km} \mathrm{~s}^{-1}\right)\end{array}$ & $\begin{array}{c}\text { rms } \\
(\mathrm{Jy} / \text { beam })\end{array}$ \\
\hline $\mathrm{C}^{34} \mathrm{~S} 2 \rightarrow 1$ & 96.44 & $\mathrm{BCD}$ & $4.21 \times 3.07$ & 66 & 0.5 & 0.01 \\
$\mathrm{CH}_{3} \mathrm{OH} 2_{k} \rightarrow 1_{k} v_{t}=1$ & 96.51 & $\mathrm{BCD}$ & $4.21 \times 3.06$ & 66 & 0.5 & 0.01 \\
$\mathrm{CH}_{3} \mathrm{OH} 2_{k} \rightarrow 1_{k} v_{t}=1$ & 96.51 & $\mathrm{AB}$ & $1.85 \times 1.36$ & 26 & 1.0 & 0.004 \\
$\mathrm{CH}_{3} \mathrm{OH} 2_{k} \rightarrow 1_{k} v_{t}=0$ & 96.72 & $\mathrm{BCD}$ & $4.19 \times 3.04$ & 65 & 8 & 0.01 \\
$\mathrm{CH}_{3} \mathrm{CN} 13_{k} \rightarrow 12_{k}$ & 238.90 & $\mathrm{BCD}$ & $2.12 \times 1.26$ & 77 & 0.8 & 0.03 \\
$\mathrm{C}^{34} \mathrm{~S} \rightarrow 4$ & 241.08 & $\mathrm{AB}$ & $0.77 \times 0.55$ & 13 & 1.5 & 0.01 \\
$\mathrm{CH}_{3} \mathrm{OH} 5_{k} \rightarrow 4_{k} v_{t}=1$ & 241.30 & $\mathrm{AB}$ & $0.77 \times 0.55$ & 13 & 1.5 & 0.01 \\
$\mathrm{CH}_{3} \mathrm{OH} 5_{k} \rightarrow 4_{k} v_{t}=0$ & 241.85 & $\mathrm{BCD}$ & $2.58 \times 1.35$ & 80 & 0.8 & 0.03 \\
$\mathrm{H}_{2} \mathrm{CS} 7_{1,6} \rightarrow 6_{1,5}$ & 243.98 & $\mathrm{AB}$ & $0.77 \times 0.55$ & 8 & 1.5 & 0.01 \\
$\mathrm{SO}_{2} 14_{0,14} \rightarrow 13_{1,13}$ & 244.25 & $\mathrm{AB}$ & $0.77 \times 0.55$ & 8 & 1.5 & 0.01 \\
$\mathrm{CH}_{3} \mathrm{OH} 7_{k} \rightarrow 6_{k} v_{t}=0,1$ & 338.72 & comp.-ext. & $1.93 \times 1.14$ & 86 & 1.0 & 0.1 \\
\hline
\end{tabular}

with a $v_{\text {lsr }}=-17.6 \mathrm{~km} \mathrm{~s}^{-1}$. Bandpass calibration was done with Jupiter, Uranus, Callisto, and 3C 279. We used Callisto and $3 \mathrm{C} 279$ for the flux calibration which is estimated to be accurate within $20 \%$. Phase and amplitude calibration was done via frequent observations of the quasar 3C 111 about $16.3^{\circ}$ from the phase centre. The zenith opacities, measured with the NRAO tipping radiometer located at the Caltech Submillimeter Observatory, were good during both tracks with $\tau(348 \mathrm{GHz}) \sim 0.18$ (scaled from the $225 \mathrm{GHz}$ measurement via $\tau(348 \mathrm{GHz}) \sim 2.8 \times \tau(225 \mathrm{GHz}))$. The receiver operated in a double-sided band mode with an intermediate frequency of 4-6 GHz so that the upper and lower side band were separated by $10 \mathrm{GHz}$. The correlator had a bandwidth of $2 \mathrm{GHz}$ and the channel separation was $0.8125 \mathrm{MHz}$. Measured double-sided band system temperatures corrected to the top of the atmosphere were between 150 and $500 \mathrm{~K}$, mainly depending on the elevation of the source. Details on the observational setup are given in Table 2.

The initial flagging and calibration was done with the IDL superset MIR originally developed for the Owens Valley Radio Observatory and adapted for the SMA ${ }^{5}$. The imaging and data analysis was conducted in MIRIAD (Sault et al. 1995) and MAPPING. During the observations on November 11th, 2004, the position of the primary calibrator $3 \mathrm{C} 111$ was wrong in the catalogue by $\sim 0.6^{\prime \prime}$. Therefore, we self-calibrated our secondary phase calibrator $0552+398\left(16.3^{\circ}\right.$ from the source $)$ shifting it in the map to the correct position. The solutions were then applied to IRAS $05358+3543$.

5 The MIR cookbook by Charlie Qi can be found at http://cfa-www.harvard.edu/ cqi/mircook.html 


\subsection{Single-dish observations with the IRAM 30 m telescope}

In addition to the high resolution data, we mapped an area of $70^{\prime \prime} \times 180^{\prime \prime}$ in the $5_{k} \rightarrow 4_{k} v_{t}=0 \mathrm{CH}_{3} \mathrm{OH}$ band, with the HERA receiver (Schuster et al. 2004) at the IRAM $30 \mathrm{~m}$ telescope in on-the-fly mode. The observations were performed in service-mode in February 2005, under excellent weather conditions (0.6-1.1 $\mathrm{mm}$ precipitable water vapour). The pointing was checked on Saturn and on a nearby source $(0439+360)$ and was found to be accurate to $\sim 6^{\prime \prime}$. Conversion from antenna temperature to main-beam brightness temperature was performed by using a beam efficiency of $0.48^{6}$. The beam of the $30 \mathrm{~m}$ telescope at $241.8 \mathrm{GHz}$ is $\sim 10.2^{\prime \prime}$. Unfortunately, the overlapping in the UV plane of our PdBI and $30 \mathrm{~m}$ data is poor, since the PdBI baselines start only only at $20 \mathrm{~m}$, and we were not able to combine the two datasets and recover the short spacing information. The IRAM $30 \mathrm{~m}$ data are, however, used in the following discussion to study the extended structure of the methanol emission.

\section{Observational results}

In this section we present the results of the line observations performed towards IRAS $05358+3543$. Since the observations were aimed at observing several bands of methanol transitions, the majority of detected lines comes from this molecule. However, emission from other molecular species with transitions close in frequency space to methanol is also detected. All detected lines are listed in Tables 3 (for $\mathrm{CH}_{3} \mathrm{OH}$ ) and 4 (for the other molecular species); the dust condensations where the lines are detected are also reported in the tables.

The large structured emission is sampled by the single dish methanol data, while the interferometric datasets allow us to zoom in on the gas around the protostars. These data range in angular resolution from $4^{\prime \prime}$ to $0.6^{\prime \prime}$, corresponding to 7200 and $1100 \mathrm{AU}$, respectively. Another property of these data is that a broad range of structures are filtered out from the observations, due to the missing short-spacing flux, from $\sim 25^{\prime \prime}$ in the $\mathrm{BCD}$ configuration of the PdBI at $3 \mathrm{~mm}$ to $\sim 6^{\prime \prime}$ in its AB configuration at $1.3 \mathrm{~mm}$. This means that the various datasets are differently affected by the problem, and the comparison of transitions from different configurations is not straightforward. Moreover, the UV coverage is poorly sampled also at intermediate scales, and smaller structures can be filtered out. Comparing the single dish data for the $5_{k} \rightarrow 4_{k} v_{t}=0$ band of $\mathrm{CH}_{3} \mathrm{OH}$ to the interferometric observation confirms that the PdBI data are missing fluxes. Although we cannot perform the same comparison for the rest of the dataset, negative features due to missing short spacings, heavily corrupt the other low energy line datacubes. Thus, the interpretation of the line interferometric data is potentially affected by missing flux problems.

Although our observations are among the highest spatial resolution studies of high-mass star forming regions existing today, there are obviously still limitations to what they resolve. We know from other observations that multiplicity on smaller scales happens in star forming regions of all masses (e.g., Weigelt et al. 1999; Megeath et al. 2005). The highest linear resolution of our observations $(\sim 1100 \mathrm{AU})$ is reached only in one dataset, while the typical resolution of the other data is poorer. Within our own data, the two sources with the smallest separation, mmlb and $\mathrm{mm} 1 \mathrm{a}$, are spatially resolved only in one dataset. Therefore, we cannot exclude that higher resolution would reveal more objects,

\footnotetext{
${ }^{6}$ http://www.iram.fr/IRAMES/
}

Table 3. Table of $\mathrm{CH}_{3} \mathrm{OH}$ detected transitions. In Col. $4, \star$ is used to indicate the lines with extended emission; (?) for tentative detections.

\begin{tabular}{|c|c|c|c|}
\hline Transition & $\begin{array}{c}\text { Rest frequency } \\
(\mathrm{GHz})\end{array}$ & $\begin{array}{l}E_{\text {upper }} \\
(\mathrm{K})\end{array}$ & Detections $^{a}$ \\
\hline$E 2_{1} \rightarrow 1_{1} v_{t}=1$ & 96.492 & 298 & $1 \mathrm{a}$ \\
\hline$E 2_{0} \rightarrow 1_{0} v_{t}=1$ & 96.493 & 308 & $1 \mathrm{a}$ \\
\hline$A 2_{0} \rightarrow 1_{0} v_{t}=1$ & 96.514 & 431 & $1 \mathrm{a}(?)$ \\
\hline$E 2_{-1} \rightarrow 1_{-1} v_{t}=0$ & $96.739^{b}$ & 13 & $1,2,3$ \\
\hline$A 2_{0} \rightarrow 1_{0} v_{t}=0$ & $96.741^{b}$ & 7 & $1,2,3$ \\
\hline$E 2_{0} \rightarrow 1_{0} v_{t}=0$ & $96.745^{b}$ & 20 & $1,2,3$ \\
\hline$E 2_{1} \rightarrow 1_{1} v_{t}=0$ & $96.756^{b}$ & 28 & $1,2,3$ \\
\hline$E 22_{-6} \rightarrow 23_{-5} v_{t}=0$ & 241.043 & 776 & $1 \mathrm{a}(?)$ \\
\hline$E 5_{3} \rightarrow 4_{3} v_{t}=1$ & 241.167 & 452 & $1 \mathrm{a}$ \\
\hline$A 5_{ \pm 4} \rightarrow 4_{ \pm 4} v_{t}=1$ & 241.178 & 516 & $1 \mathrm{a}$ \\
\hline$E 5_{-3} \rightarrow 4_{-3} v_{t}=1$ & 241.180 & 357 & $1 \mathrm{a}$ \\
\hline$E 5_{-4} \rightarrow 4_{-4} v_{t}=1$ & 241.184 & 440 & $1 \mathrm{a}$ \\
\hline$E 5_{-2} \rightarrow 4_{-2} v_{t}=1$ & 241.187 & 399 & $1 \mathrm{a}$ \\
\hline$A 5_{2} \rightarrow 4_{2} v_{t}=1$ & 241.193 & 333 & $1 \mathrm{a}$ \\
\hline$A 5_{2} \rightarrow 4_{2} v_{t}=1$ & 241.196 & 333 & $1 \mathrm{a}$ \\
\hline$A 5_{ \pm 3} \rightarrow 4_{ \pm 3} v_{t}=1$ & 241.198 & 431 & $1 \mathrm{a}$ \\
\hline$E 5_{1} \rightarrow 4_{1} v_{t}=1$ & 241.204 & 326 & $1 \mathrm{a}$ \\
\hline$E 5_{0} \rightarrow 4_{0} v_{t}=1$ & 241.206 & 335 & $1 \mathrm{a}$ \\
\hline$E 5_{2} \rightarrow 4_{2} v_{t}=1$ & 241.211 & 435 & $1 \mathrm{a}$ \\
\hline$E 5_{-1} \rightarrow 4_{-1} v_{t}=1$ & 241.238 & 448 & $1 \mathrm{a}$ \\
\hline$A 5_{0} \rightarrow 4_{0} v_{t}=1$ & 241.268 & 458 & $1 \mathrm{a}$ \\
\hline$A 5_{1} \rightarrow 4_{1} v_{t}=1$ & 241.441 & 360 & $1 \mathrm{a}$ \\
\hline$E 5_{0} \rightarrow 4_{0} v_{t}=0$ & 241.700 & 48 & $1,2,3$, line, ${ }^{\star}$ \\
\hline$E 5_{-1} \rightarrow 4_{-1} v_{t}=0$ & 241.767 & 40 & $1,2,3$, line, ${ }^{\star}$ \\
\hline$A 5_{0} \rightarrow 4_{0} v_{t}=0$ & 241.791 & 34 & $1,2,3$, line, ${ }^{\star}$ \\
\hline$A 5_{ \pm 4} \rightarrow 4_{ \pm 4} v_{t}=0$ & 241.807 & 115 & 1,2, line \\
\hline$E 5_{-4} \rightarrow 4_{-4} v_{t}=0$ & 241.813 & 123 & 1,2 , line \\
\hline$E 5_{4} \rightarrow 4_{4} v_{t}=0$ & 241.830 & 131 & 1,2 , line \\
\hline$A 5_{ \pm 3} \rightarrow 4_{ \pm 3} v_{t}=0$ & 241.833 & 85 & 1,2 , line \\
\hline$A 5_{2} \rightarrow 4_{2} v_{t}=0$ & $241.842^{c}$ & 73 & 1,2 , line \\
\hline$E 5_{3} \rightarrow 4_{3} v_{t}=0$ & $241.844^{c}$ & 83 & 1,2 , line \\
\hline$E 5_{-3} \rightarrow 4_{-3} v_{t}=0$ & 241.852 & 98 & 1,2, line \\
\hline$E 5_{1} \rightarrow 4_{1} v_{t}=0$ & 241.879 & 56 & $1,2,3(?)$, line \\
\hline$A 5_{2} \rightarrow 4_{2} v_{t}=0$ & 241.888 & 73 & 1,2, line \\
\hline$E 5_{-2} \rightarrow 4_{-2} v_{t}=0$ & $241.904^{c}$ & 61 & $1,2,3$, line, ${ }^{\star}$ \\
\hline$E 5_{2} \rightarrow 4_{2} v_{t}=0$ & $241.905^{c}$ & 57 & $1,2,3$, line, * \\
\hline$A 5_{1} \rightarrow 4_{1} v_{t}=0$ & 243.916 & 40 & 1,2, line, ${ }^{\star}$ \\
\hline$E 7_{-2} \rightarrow 6_{-2, v=1}$ & 337.605 & 429 & $1 \mathrm{a}$ \\
\hline$A 7_{2} \rightarrow 6_{2} v_{t}=1$ & 337.636 & 363 & $1 \mathrm{a}$ \\
\hline$E 7_{-1} \rightarrow 6_{-1} v_{t}=1$ & 337.642 & 356 & $1 \mathrm{a}$ \\
\hline$E 7_{0} \rightarrow 6_{0} v_{t}=1$ & 337.644 & 365 & $1 \mathrm{a}$ \\
\hline$E 7_{-4} \rightarrow 6_{-4} v_{t}=1$ & 337.646 & 470 & $1 \mathrm{a}$ \\
\hline$E 7_{0} \rightarrow 6_{0} v_{t}=0$ & 338.125 & 78 & 1,2, line, ${ }^{\star}$ \\
\hline$E 7_{-1} \rightarrow 6_{-1} v_{t}=0$ & 338.345 & 71 & $1,2,3(?)$, line, ^ \\
\hline$E 7_{6} \rightarrow 6_{6} v_{t}=0$ & 338.405 & 244 & 1 \\
\hline$A 7_{0} \rightarrow 6_{0} v_{t}=0$ & 338.409 & 65 & $1,2,3(?)$, line, ${ }^{\star}$ \\
\hline$A 7_{ \pm 5} \rightarrow 6_{ \pm 5} v_{t}=0$ & 338.486 & 203 & 1 \\
\hline$A 7_{ \pm 4} \rightarrow 6_{ \pm 4} v_{t}=0$ & $338.513^{c}$ & 145 & 1,2 , line \\
\hline$A 7_{2} \rightarrow 6_{2} v_{t}=0$ & $338.513^{c}$ & 103 & 1,2 , line \\
\hline$E 7_{4} \rightarrow 6_{4} v_{t}=0$ & 338.530 & 161 & 1 \\
\hline$A 7_{3} \rightarrow 6_{3} v_{t}=0$ & $338.541^{c}$ & 115 & 1,2 , line \\
\hline$A 7_{3} \rightarrow 6_{3} v_{t}=0$ & $338.543^{c}$ & 115 & 1,2 , line \\
\hline$E 7_{-3} \rightarrow 6_{-3} v_{t}=0$ & 338.560 & 128 & 1 \\
\hline$E 7_{3} \rightarrow 6_{3} v_{t}=0$ & 338.583 & 118 & 1,2 , line \\
\hline$E 7_{1} \rightarrow 6_{1} v_{t}=0$ & $338.615^{d}$ & 86 & 1,2 , line \\
\hline$A 7_{2} \rightarrow 6_{2} v_{t}=0$ & 338.640 & 103 & 1,2, line \\
\hline$E 7_{2} \rightarrow 6_{2} v_{t}=0$ & $338.722^{c}$ & 87 & 1,2, line ${ }^{\star}$ \\
\hline$E 7_{-2} \rightarrow 6_{-2} v_{t}=0$ & $338.723^{c}$ & 91 & 1,2 , line, ${ }^{\star}$ \\
\hline
\end{tabular}

${ }^{a} 1,2,3$ indicate $\mathrm{mm} 1, \mathrm{~mm} 2$, and $\mathrm{mm} 3$, respectively. 1 is used for data where $\mathrm{mmla}$ and $\mathrm{mm} 1 \mathrm{~b}$ are not resolved; line is used for the position $\left(1.2^{\prime \prime}, 0.6^{\prime \prime}\right)$ from $\mathrm{mm} 2$.

${ }^{b}$ The $2_{k} \rightarrow 1_{k}$ quartet of lines is unresolved in velocity.

${ }^{c}$ Blend of lines.

${ }^{d}$ Blend with the $20_{1,19} \rightarrow 19_{2,18} \mathrm{SO}_{2}$ line at $338.612 \mathrm{GHz}$. 
Table 4. Table of observed transitions from other molecular species. In Col. $4, \star$ is used to indicate the lines with extended emission.

\begin{tabular}{|c|c|c|c|}
\hline Transition & $\begin{array}{c}\text { Rest frequency } \\
(\mathrm{GHz})\end{array}$ & $\begin{array}{l}E_{\text {upper }} \\
(\mathrm{K})\end{array}$ & Detections $^{a}$ \\
\hline $\mathrm{C}^{34} \mathrm{~S} 2 \rightarrow 1$ & 96.413 & 7 & $1,2,3, \star$ \\
\hline $\mathrm{C}^{34} \mathrm{~S} 5 \rightarrow 4$ & 241.016 & 35 & 1 \\
\hline $\mathrm{CH}_{3} \mathrm{CN} 13_{6} \rightarrow 12_{6}$ & 238.972 & 337 & 1 \\
\hline $\mathrm{CH}_{3} \mathrm{CN} 13_{5} \rightarrow 12_{5}$ & 239.022 & 259 & 1 \\
\hline $\mathrm{CH}_{3} \mathrm{CN} 13_{4} \rightarrow 12_{4}$ & 239.065 & 195 & 1 \\
\hline $\mathrm{CH}_{3} \mathrm{CN} 13_{3} \rightarrow 12_{3}$ & 239.096 & 145 & 1 , line \\
\hline $\mathrm{CH}_{3} \mathrm{CN} 13_{2} \rightarrow 12_{2}$ & 239.119 & 109 & 1 \\
\hline $\mathrm{CH}_{3} \mathrm{CN} 13_{1} \rightarrow 12_{1}$ & 239.133 & 87 & 1 , line \\
\hline $\mathrm{CH}_{3} \mathrm{CN} 13_{0} \rightarrow 12_{0}$ & 239.138 & 80 & 1 , line \\
\hline HDO $2_{1,1} \rightarrow 2_{1,2}$ & 241.561 & 95 & 1 \\
\hline $\mathrm{SO}_{2} 5_{2,4} \rightarrow 4_{1,3}$ & 241.616 & 24 & 1 , line \\
\hline $\mathrm{HNCO} 11_{2,10} \rightarrow 10_{2,9}$ & 241.704 & 243 & 1 \\
\hline $\mathrm{HNCO} 11_{2,9} \rightarrow 10_{2,8}$ & 241.708 & 243 & 1 \\
\hline $\mathrm{HNCO} 11_{0,11} \rightarrow 10_{0,10}$ & 241.774 & 70 & 1 \\
\hline $\mathrm{H}_{2} \mathrm{CS} 7_{1,6} \rightarrow 6_{1,5}$ & 244.048 & 60 & 1 \\
\hline $\mathrm{SO}_{2} 14_{0,14} \rightarrow 13_{1,13}$ & 244.254 & 94 & 1 \\
\hline $\mathrm{C}^{34} \mathrm{~S} 7 \rightarrow 6$ & 337.396 & 65 & 1 , line \\
\hline $\mathrm{H}_{2} \mathrm{CS} 10_{1,10} \rightarrow 9_{1,9}$ & 338.083 & 102 & 1 \\
\hline $\mathrm{SO}_{2} 18_{4,14} \rightarrow 18_{3,15}$ & 338.306 & 197 & 1 \\
\hline $\mathrm{SO}_{2} 20_{1,19} \rightarrow 19_{2,18}$ & $338.612^{b}$ & 199 & 1 \\
\hline
\end{tabular}

a $1,2,3$ indicate $\mathrm{mm} 1, \mathrm{~mm} 2$, and $\mathrm{mm} 3$, respectively. 1 is used for data where $\mathrm{mmla}$ and $\mathrm{mm} / \mathrm{b}$ are not resolved; line is used for the position $\left(1.2^{\prime \prime}, 0.6^{\prime \prime}\right)$ off of $\mathrm{mm} 2$.

${ }^{b}$ Blend with the $7_{1} \rightarrow 6_{1} v_{t}=0 \mathrm{CH}_{3} \mathrm{OH}-E$ line at $338.615 \mathrm{GHz}$.

and that the parameters we derive do not sample the gas around a single protostar. However, if this was the case, the multiple unresolved sources would form from the same reservoir of dust and gas. Assuming that they are gravitationally bound, the parameters derived in our current study would still be relevant to the analysis of the collapsing cores.

\subsection{Extended emission}

Emission from relatively low excitation lines is extended, as shown in Fig. 1a, where the integrated intensity of the $5_{0} \rightarrow$ $4_{0}, v_{t}=0 \mathrm{CH}_{3} \mathrm{OH}-A$ transition taken with the $30 \mathrm{~m}$ telescope is presented.

For methanol, the emission at the cloud velocity shows a distribution very similar to the continuum emission. The offset of the line emission peak from the continuum peak is real, since it was already observed in methanol and in other molecular species (Beuther et al. 2002a). In their single-dish observations, Beuther et al. (2002a) detected red and blue non-Gaussian emission in the $5_{k} \rightarrow 4_{k}$ methanol band, and suggested that both emissions are associated with a third outflow in the region to the west of the continuum peak. Their results are confirmed by our data, which show a bipolar distribution in the low excitation transitions of $\mathrm{CH}_{3} \mathrm{OH}$ (Fig. 1a) with a better signal-to-noise ratio. Within the pointing uncertainties and the low resolution of the data, the centre of this outflow seems to be associated with one of the $\mathrm{H}^{13} \mathrm{CO}^{+}$ peaks (indicated by a pentagon in Fig. 1a) detected by Beuther et al. (2002a). If this were the case, our observations would solve the problem of the powering source of the third outflow of the region ( $C$ in the notation of Beuther et al. 2002a), which was previously not assigned. However, higher angular resolution is needed to assign the centre of this flow beyond a doubt.

The interpretation of the interferometric data of the same transition is not as straightforward as for the single-dish data. In
Fig. 1b, the integrated intensity of the $5_{0} \rightarrow 4_{0} \mathrm{CH}_{3} \mathrm{OH}-A$ line is shown as seen in the PdBI observations, together with the direction of the two outflows originating from the vicinity of $\mathrm{mm} 1$. The region west of the dust cores, where the bipolar distribution is detected in the single-dish data, is only partially in the field of view of the PdBI observations. The intensity map is strongly affected by missing flux problems, which results in filtering out the extended emission. For this reason, the PdBI map of this transition looks clumpy. The emission is detected all over the dust continuum peaks, often red- and/or blue-shifted. These emission spots could be caused by the high velocity $\mathrm{CO}$ outflow originating from the vicinity of $\mathrm{mm} 1$ (see Fig. 1b). However, we refrain from a further discussion of these features, given the missing short spacing information of our methanol data.

The distribution of $\mathrm{C}^{34} \mathrm{~S}$ line is puzzling (Fig. 2). While the $2 \rightarrow 1$ line extends over an area of approximately $20^{\prime \prime} \times 20^{\prime \prime}$ around the main dust condensations, and the $7 \rightarrow 6$ transition is found in association with $\mathrm{mm} 1$ (although marginally detected) and towards $\mathrm{mm} 2$, the $5 \rightarrow 4$ line is detected only on mm1a and $\mathrm{mm} 1 \mathrm{~b}$ (see Fig. 2). In the $5 \rightarrow 4 \mathrm{C}^{34} \mathrm{~S}$ line, two velocity components (see discussion on $\mathrm{mm} 1$ in Sect. 3.2) are detected, one at $v_{\text {lsr }} \sim-17.6 \mathrm{~km} \mathrm{~s}^{-1}$ and the other at $v_{\mathrm{lsr}} \sim-13 \mathrm{~km} \mathrm{~s}^{-1}$, the first on mmla and the second on mmla and on the vicinity of $\mathrm{mm} 1 \mathrm{~b}$. The emission of the $5 \rightarrow 4 \mathrm{C}^{34} \mathrm{~S}$ line extends to the south-west, as does that of the $7 \rightarrow 6$ line. However, the spectra are noisy, and the line is detected with a significance level not better than $2 \sigma$. In Fig. 2, the level contours start from a value of $3 \sigma$, and the feature on the south-west of mmla is not seen in the $5 \rightarrow 4$ transition.

Olmi \& Cesaroni (1999) studied the excitation of CS and $\mathrm{C}^{34} \mathrm{~S}$ towards a sample of ultra-compact HII regions, and found, as expected given the energies of the levels involved, that the $5 \rightarrow 4 \mathrm{C}^{34} \mathrm{~S}$ transition is usually stronger than the $7 \rightarrow 6$ line, making the non detection of the $5 \rightarrow 4$ line on $\mathrm{mm} 2$ difficult to interpret. However, this line was observed in the AB configurations of the PdB interferometer, where source structures larger than $6^{\prime \prime}$ are filtered out. Therefore, the unexpected spatial distribution of this transition, as well as its profile, can be an artifact due to the missing short spacing information.

Comparing the spatial distribution of the $2 \rightarrow 1$ and $7 \rightarrow$ $6 \mathrm{C}^{34} \mathrm{~S}$ lines, which are affected by missing flux problems on larger scales ( $\geq 30^{\prime \prime}$ and $\geq 16^{\prime \prime}$, respectively), it emerges that they do not peak on the protostellar cores, but in the gas between the cores, where the temperature most likely drops down. Observations of the hot core in G29.96-0.02 (Beuther et al. $2007 b$ ), with the same frequency setup of our SMA data, show a similar effect, with the $7 \rightarrow 6 \mathrm{C}^{34} \mathrm{~S}$ line peaked in the gas between the (sub)mm continuum peaks. On the other hand, in G29.96-0.02, the $8_{8} \rightarrow 77{ }^{34} \mathrm{SO}$ line peaks on the hot core. Since CS should desorb from dust grains relatively early in the evolution of the source (Viti et al. 2004), while the SO and $\mathrm{SO}_{2}$ abundances should increase with time, Beuther et al. (2007b) interpret the distributions of $\mathrm{C}^{34} \mathrm{~S}$ and ${ }^{34} \mathrm{SO}$ in G29.96-0.02 as an evolutionary indicator. In our case, we did not detect the $8_{8} \rightarrow 77_{7}^{34} \mathrm{SO}$ transition toward IRAS $0535+3543$. However, we observed four transitions of $\mathrm{SO}_{2}$, and all peak on the main dust condensation $\mathrm{mm} 1$ (Fig. 3). Although the evolutionary scenario outlined for G29.96-0.02 seems to be confirmed by our observations of the $2 \rightarrow 1$ and $7 \rightarrow 6 \mathrm{C}^{34} \mathrm{~S}$, and of the $\mathrm{SO}_{2}$ transitions, the distribution of the $5 \rightarrow 4 \mathrm{C}^{34} \mathrm{~S}$ line does not find an easy interpretation within this theory.

Similarly to $\mathrm{C}^{34} \mathrm{~S}$, also $\mathrm{H}_{2} \mathrm{CS}$ shows different morphologies in the two transitions in our datasets (Fig. 3), with the $10_{1,10} \rightarrow 9_{1,9}$ line $\left(E_{\text {up }} \sim 100 \mathrm{~K}\right)$ having a second peak at an 

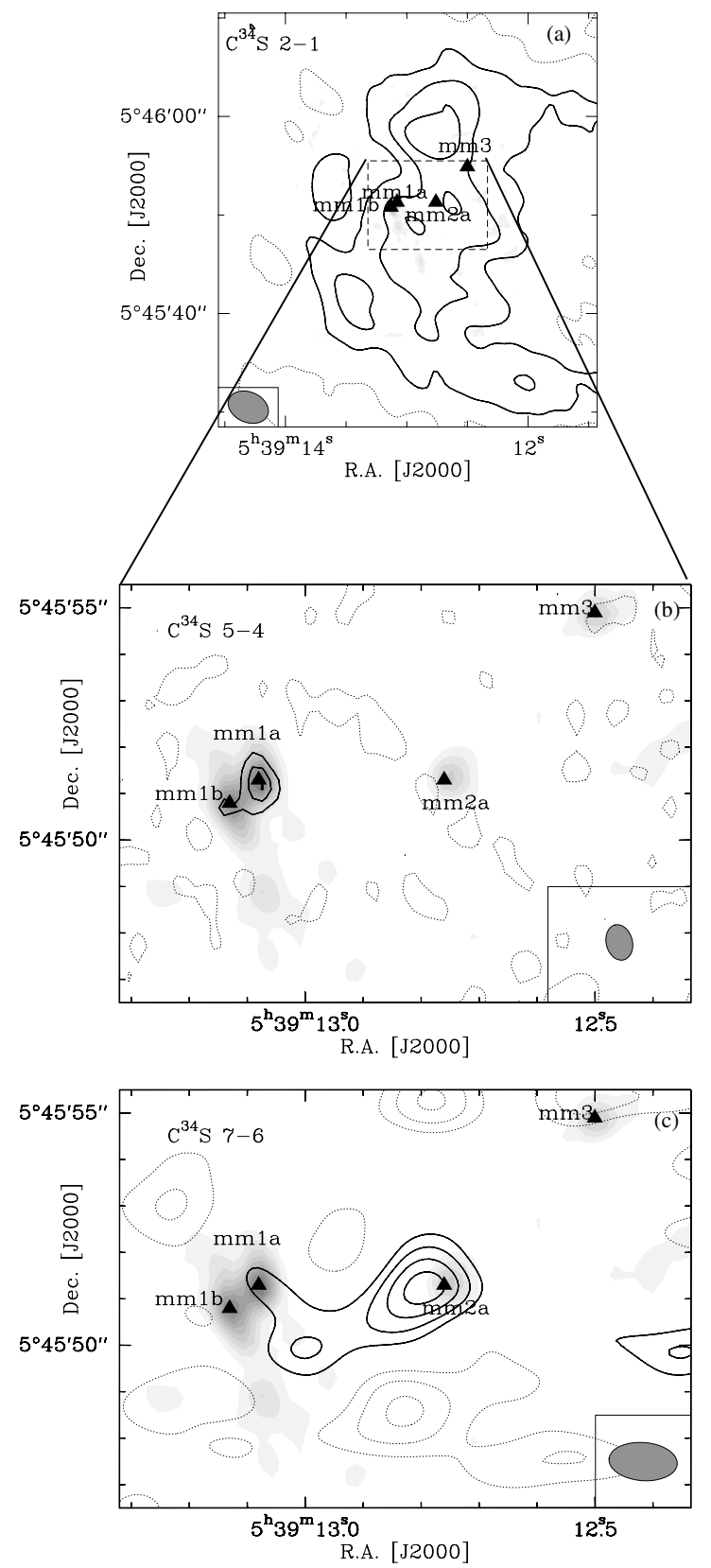

Fig. 2. In grey scale the $1.3 \mathrm{~mm}$ continuum emission (from the $A B$ configuration of the Plateau de Bure Interferometer). The black contours show the integrated line intensity of the $2 \rightarrow 1 \mathbf{a}$ ), the $5 \rightarrow 4 \mathbf{b}$ ), and of the $7 \rightarrow 6 \mathrm{C}^{34} \mathrm{~S}$ lines c). Level contours are from $0.1 \mathrm{Jy}_{\text {beam }}{ }^{-1} \mathrm{~km} \mathrm{~s}^{-1}$ in steps of 0.2 for the $2 \rightarrow 1$ transition; from $0.3 \mathrm{Jy}_{\text {beam }}{ }^{-1} \mathrm{~km} \mathrm{~s}^{-1}$ in steps of 0.3 for the $5 \rightarrow 4$ line, and from 1.5 in steps of 0.5 for the $7 \rightarrow 6$. Level contours for the continuum are in step of 0.003 from $0.003 \mathrm{Jy}$ beam ${ }^{-1}$. The dotted contours show the negative emission $\left(-0.1 \mathrm{Jy}\right.$ beam ${ }^{-1} \mathrm{~km} \mathrm{~s}^{-1}$ for the $2 \rightarrow 1$ and $5 \rightarrow 4$ lines; from -1.5 in step of $0.5 \mathrm{Jy} \mathrm{beam}^{-1} \mathrm{~km} \mathrm{~s}^{-1}$ for the $7 \rightarrow 6$ transition). The dashed lines in panel a) outline the region shown in the other two panels. The different beams are also given.

offset position from $\mathrm{mm} 2$ (see the discussion below), while the $7_{1,6} \rightarrow 6_{1,5}$ is found only on mm1a, despite its lower level energy $\left(E_{\text {up }} \sim 60 \mathrm{~K}\right)$. We believe that these data are heavily contaminated by missing flux problems at intermediate and short scales, and refrain from any analysis of this molecular species.

\subsection{Molecular emission from the dust cores}

The molecular spectra of the three main dust condensations, $\mathrm{mm} 1, \mathrm{~mm} 2$ and $\mathrm{mm} 3$, differ significantly from each other, reflecting the different physical conditions, and probably time evolution, of the dust cores. Maps of the integrated intensity of several transitions are shown in Figs. 3 and 4. All high excitation lines peak on $\mathrm{mm} 1$, and specifically on mmla for the dataset where the cores mm $1 \mathrm{a}$ and $\mathrm{mm} 1 \mathrm{~b}$ are spatially resolved. However, several moderately excited $\left(E_{\text {up }} \leq 130 \mathrm{~K}\right)$ methanol lines are extended towards the second dust core $\mathrm{mm} 2$, and show a peak of intensity at $\left(1.2^{\prime \prime}, 0.6^{\prime \prime}\right)$ from $\mathrm{mm} 2$.

Source mm1: Fig. 5 presents the observed spectral bands at 238, 241 and $338 \mathrm{GHz}$, respectively, towards the main dust condensation $\mathrm{mm} 1$. High excitation $\left(E_{\text {up }}>200 \mathrm{~K}\right)$ transitions are detected towards this core; however, the datasets where the two dust condensations mmla and $\mathrm{mm} 1 \mathrm{~b}$ are resolved, show that the emission from high excited lines is associated only with mmla. Only the $5 \rightarrow 4 \mathrm{C}^{34} \mathrm{~S}$ transition $\left(v \sim-13.4 \mathrm{~km} \mathrm{~s}^{-1}\right)$ is detected on mm1b. The lack of molecular emission from mm1b could be a bias of the observations: the two dusts condensations are resolved only in the $\mathrm{AB}$ configuration of the PdBI, which was aimed at the detection of torsionally excited lines of methanol and had the $5 \rightarrow 4 \mathrm{C}^{34} \mathrm{~S}$ as the only low excitation transition. The two condensations have very similar properties for the continuum emission (Beuther et al. 2007a). However, other observations suggest that $\mathrm{mm} 1 \mathrm{a}$ and $\mathrm{mmlb}$ are indeed of different nature, as methanol class II masers (Minier et al. 2000), and a mid-infrared source (Longmore et al. 2006) are detected on mmla, but not on mm1b. A similar chemical differentiation of two cores of very close masses was recently found by Zhang et al. (2007) in the high-mass (proto)stellar cluster AFGL5142. As suggested for AFGL5142, a possible interpretation of our observations is that $\mathrm{mm} 1 \mathrm{~b}$ is in an earlier evolutionary phase than $\mathrm{mm} 1 \mathrm{a}$, still not characterised by a rich chemistry.

Source mmla: several methanol transitions, in the ground state as well as in the first torsionally excited level, show two velocity components. The same profile is found in the $12_{k} \rightarrow 11_{k}$ $\mathrm{CH}_{3} \mathrm{CN}$ band (SMA data, Beuther priv. comm.) and in the $13_{k} \rightarrow$ $12_{k}$ lines, in the $7_{1,6} \rightarrow 6_{1,5} \mathrm{H}_{2} \mathrm{CS}$ line, and in the $5 \rightarrow 4 \mathrm{C}^{34} \mathrm{~S}$ transition. HNCO is also showing the same behaviour in the the $11_{0,11} \rightarrow 10_{0,10}$ line at $\sim-17.8$ and $\sim-15.3 \mathrm{~km} \mathrm{~s}^{-1}$. Two peaks are also detected in the $11_{2,9} \rightarrow 10_{2,8}$ transition, but the blend with the $5_{0} \rightarrow 4_{0} \mathrm{CH}_{3} \mathrm{OH}-E$ line and with the $11_{2,10} \rightarrow$ $10_{2,9}$ transition complicates the interpretation of this profile. On the other hand, the $\mathrm{SO}_{2}$ and $\mathrm{HDO}$ lines at $\sim 241.6 \mathrm{GHz}$ show only one velocity component, peaked at -16.6 and $-14.9 \mathrm{~km} \mathrm{~s}^{-1}$, respectively. For the HDO line, this corresponds to the velocity in between the two peaks detected in the other transitions. An example of the double peak profile of mmla is given in Fig. 6, where part of the $5_{k} \rightarrow 4_{k}, v_{t}=1 \mathrm{CH}_{3} \mathrm{OH}$ band is shown. For the $7_{1,6} \rightarrow 6_{1,5} \mathrm{H}_{2} \mathrm{CS}$ and the $5 \rightarrow 4 \mathrm{C}^{34} \mathrm{~S}$ lines, this profile could be caused by missing flux; self absorption or missing flux problems could affect the low energy transitions of methanol. The hypothesis of self absorption is strengthened by the profile of HDO transition. However, since the double-peaked profile is found also at high energies, where the optical depth is unlikely to be high, even in rare molecular species, we believe that the two peaks are, at least for the torsionally excited lines of $\mathrm{CH}_{3} \mathrm{OH}$, for $\mathrm{CH}_{3} \mathrm{CN}$ and $\mathrm{HNCO}$, real, and not due to self absorption. In several cases, overlap between different lines complicates the identification of the two components; however, a few lines $\left(5_{0} \rightarrow 4_{0}\right.$ and $5_{-1} \rightarrow 4_{-1} v_{t}=1 \mathrm{CH}_{3} \mathrm{OH} ; 13_{4} \rightarrow 12_{4} \mathrm{CH}_{3} \mathrm{CN}$ ) do not overlap with any other transitions. By Gauss fitting their line profiles, 


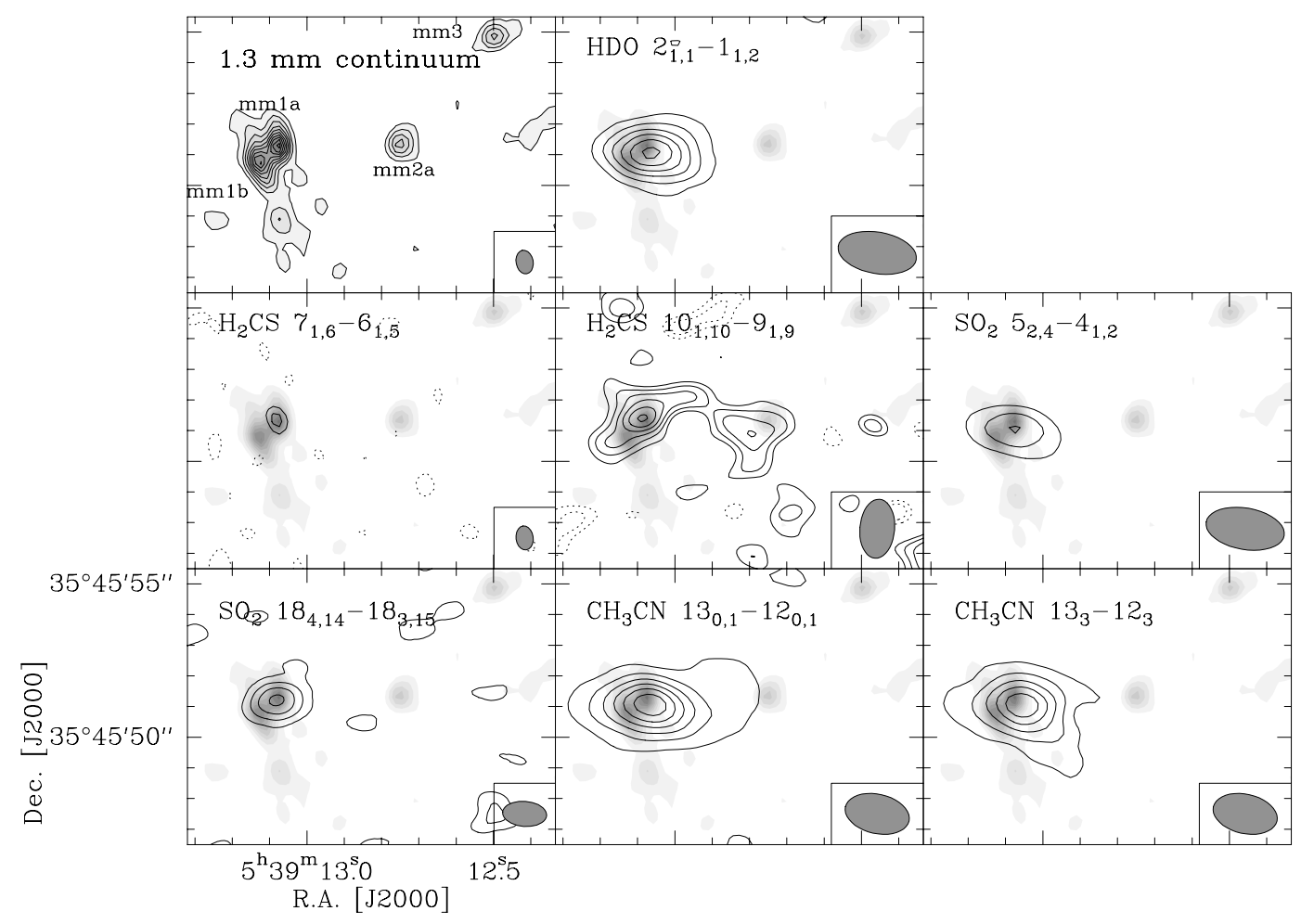

Fig. 3. Compilation of integrated intensity maps of transitions from several molecular species, shown as black contours. In grey scale (and in the solid contours in the first panel) the $1.3 \mathrm{~mm}$ continuum emission from the AB configuration of the Plateau de Bure Interferometer. Level contours for the line images are from $0.5 \mathrm{Jy} \mathrm{beam}^{-1} \mathrm{~km} \mathrm{~s}^{-1}$, in step of 0.3 ; for the $7_{1,6} \rightarrow 6_{1,5} \mathrm{H}_{2} \mathrm{CS}$ transition, from $0.3 \mathrm{Jy} \mathrm{beam}^{-1} \mathrm{~km} \mathrm{~s}^{-1}$, and from $1 \mathrm{Jy} \mathrm{beam}^{-1} \mathrm{~km} \mathrm{~s}^{-1}$ for the $18_{4,14} \rightarrow 18_{3,15} \mathrm{SO}_{2}$ line. Level contours for the $13_{0,1} \rightarrow 12_{0,1} \mathrm{CH}_{3} \mathrm{CN}$ transitions are in step of $0.6 \mathrm{Jy} \mathrm{beam}^{-1} \mathrm{~km} \mathrm{~s}^{-1}$. The dotted contours show the negative emission: for the $7_{1,6} \rightarrow 6_{1,5} \mathrm{H}_{2} \mathrm{CS}$ transition $-0.1 \mathrm{Jy}$ beam ${ }^{-1} \mathrm{~km} \mathrm{~s}^{-1}$; for the $10_{1,10} \rightarrow 9_{1,9} \mathrm{H}_{2} \mathrm{CS}$ line -1.5 and $-1.2 \mathrm{Jy}_{\text {beam }}{ }^{-1} \mathrm{~km} \mathrm{~s}^{-1}$. Continuum contours are the same as in Fig. 2. The beam is shown in the right bottom corner.

we derive linewidths of $\sim 3$ and $\sim 4 . \mathrm{km} \mathrm{s}^{-1}$, and system velocities of $\sim-17.6 \mathrm{~km} \mathrm{~s}^{-1}$ and $\sim-11.6 \mathrm{~km} \mathrm{~s}^{-1}$, respectively. Both velocity components peak on mmla.

We fitted the peak position of each velocity channels of the $5_{-1} \rightarrow 4_{-1} v_{t}=1 \mathrm{CH}_{3} \mathrm{OH}-E$ line, and detected a velocity gradient along an elongated structure (Fig. 7a). This linear structure seems to be shifted from the millimetre continuum peak. The same velocity gradient of the $v_{t}=1$ methanol lines is found in the $19 \rightarrow 18$ OCS line at $231 \mathrm{GHz}$ (detected with the SMA, Beuther priv. comm.), and in the $6.7 \mathrm{GHz}$ methanol maser transition (Minier et al. 2000). In Fig. 7a, we show for comparison the positions of the velocity channels of the maser line, and in Fig. $7 \mathrm{~b}$ the first moment map of the $19 \rightarrow 18$ OCS transition. Moreover, observations with the SMA with an angular resolution of $\sim 3^{\prime \prime}$ (Beuther, priv. comm) show that the $2 \rightarrow 1 \mathrm{C}^{18} \mathrm{O}$ line is associated with the high velocity outflow of Beuther et al. (2002a), and has a velocity gradient perpendicular to the linear structure detected in methanol. While an unresolved expanding or contracting shell of gas would also produce the double-peaked profile detected towards $\mathrm{mm} 1 \mathrm{a}$, the linear velocity gradient detected in methanol and OCS cannot be explained by these scenarios.

The line profile detected towards mmla is reminiscent of the double-peaked profiles found in low and intermediate mass tilted circumstellar disks, in optically thin and thick lines (Beckwith \& Sargent 1993). Moreover, the double-peaked profile arises from a region $(R \leq 550 \mathrm{AU})$ comparable in size to the candidate disks surrounding high-mass (proto)stars (for a compilation of them, see Cesaroni et al. 2007). The detection of a velocity gradient is not conclusive of a circumstellar disk. However, we believe that the velocity structure detected in methanol and perpendicular to the high-velocity outflow, together with the double-peaked profile, and the size of the emitting gas are strongly suggestive of a rotating structure in IRAS $05358-\mathrm{mm} 1 \mathrm{a}$. To investigate whether this structure is a circumstellar disk or a toroid like the ones discussed by Cesaroni et al. (2007), higher linear and velocity resolutions are needed to study the velocity field of the innermost gas around mm1a. We note, however, that toroids have typical radii of several thousands $\mathrm{AU}$, while the radius of the structure around mm1a has an upper limit of $550 \mathrm{AU}$.

Alternatively, two different hot components with a separation of less than $1100 \mathrm{AU}$ would also produce a double-peaked profile, and a velocity gradient along a linear structure. Since the two lines have very similar intensities and linewidths, the physical conditions in the two cores would be comparable (as derived in paragraph Sect. 4.1). The total mass of the binaries can be derived via (e.g., Chen et al. 2006)

$M_{\mathrm{tot}}=\frac{A \times \Delta v^{2}}{G \times \sin ^{2}(i)}$

where $A$ is the semi-major axis of the elliptical orbit of the two stars, $G$ the gravitational constant, $i$ the inclination angle, which is unknown, and $\Delta v$ the relative velocity between the two cores. An upper limit to the major axis is given by the resolution of the data (0.6", corresponding to $\sim 1100 \mathrm{AU}$, at this distance). In Sect. 4.1, the sizes of the two sources are derived by fitting the $13_{k} \rightarrow 12_{k} \mathrm{CH}_{3} \mathrm{CN}$ band. Therefore, the lower limit to the major axis of the orbit is taken as the sum of their radii, corresponding 


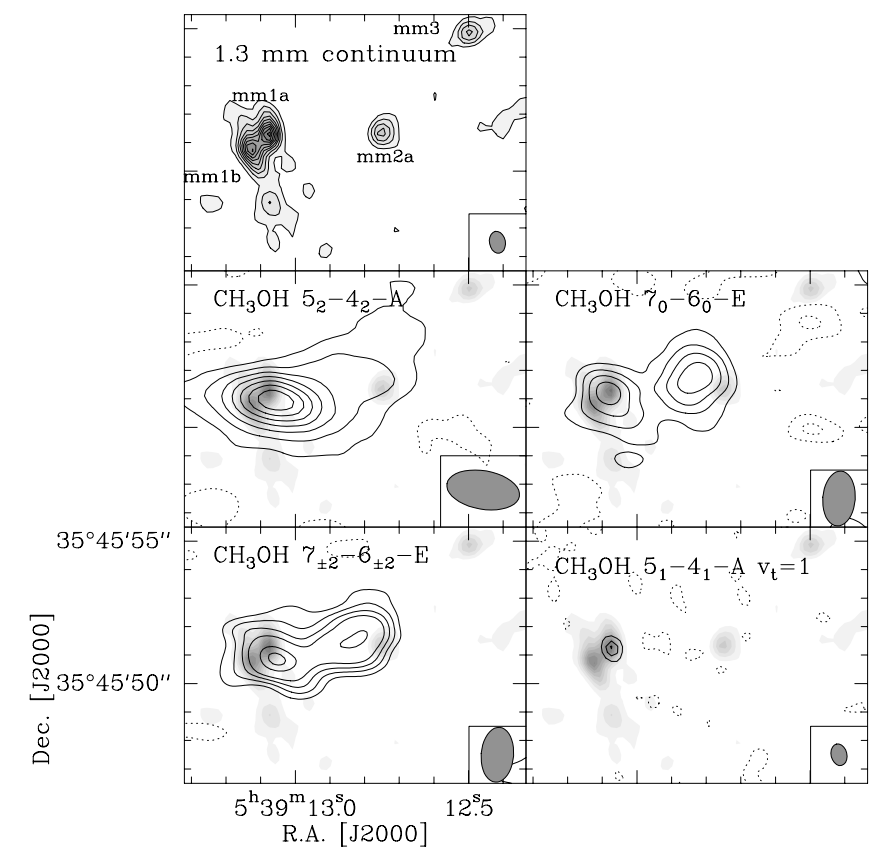

Fig. 4. Compilation of integrated intensity maps of $\mathrm{CH}_{3} \mathrm{OH}$, shown as black contours. In grey scale (and in the solid contours in the first panel) the $1.3 \mathrm{~mm}$ continuum emission from the $\mathrm{AB}$ configuration of the Plateau de Bure Interferometer. Level contours for the line images are from: $0.3 \mathrm{Jy}_{\text {beam }}{ }^{-1} \mathrm{~km} \mathrm{~s}^{-1}$ in step of $0.4,1 \mathrm{Jy}_{\text {beam }}^{-1} \mathrm{~km} \mathrm{~s}^{-1}$ in step of $1,1.5 \mathrm{Jy} \mathrm{beam}^{-1} \mathrm{~km} \mathrm{~s}^{-1}$ in step of $1,0.2 \mathrm{Jy}_{\text {beam }}{ }^{-1} \mathrm{~km} \mathrm{~s}^{-1}$ in step of 0.2 , respectively. Dotted contours are used for negative values: for the $5_{2} \rightarrow 4_{2}-A$ line $-0.2 \mathrm{Jy}_{\text {beam }}^{-1} \mathrm{~km} \mathrm{~s}^{-1} ;-2$ and $-1 \mathrm{Jy}_{\text {beam }}{ }^{-1} \mathrm{~km} \mathrm{~s}^{-1}$ for the $7_{0} \rightarrow 6_{0}-E$ line; $-2 \mathrm{Jy} \mathrm{beam}^{-1} \mathrm{~km} \mathrm{~s}^{-1}$ for the $7_{ \pm 2} \rightarrow 6_{ \pm 2}-E$ line; -0.1 for the $5_{1} \rightarrow 4_{1} \mathrm{CH}_{3} \mathrm{OH}-E v_{t}=1$ transition. Continuum contours are the same as in Fig. 2. The beam is shown in the right bottom corner.

to $0.2^{\prime \prime}$ (360 AU), which is in agreement with the extension of the linear structure in Fig. 7a. Hence, the total mass of the system ranges between 22 and $7 M_{\odot} / \sin ^{2} i$, in good agreement with the value of $13 M_{\odot}$ derived from the Lyman continuum flux (Beuther et al. 2007a).

In both cases, a rotating structure or two close-by hot cores, the single-peaked line profile of HDO would suggest that the emission is coming from a larger envelope of gas.

To discriminate between the different possibilities discussed above, higher spatial resolution observations will be required to resolve and image the system in more details. Among the current $\mathrm{cm}-\mathrm{mm}$ interferometers, only the VLA in its A configuration would allow observations with a spatial resolution significantly higher than the one of our current data. Otherwise, studies of such systems at resolutions of $0.1^{\prime \prime}$ or less in spectral lines other than $\mathrm{NH}_{3}$ will be possible in the submm windows only when the Atacama Large Millimeter Array (ALMA) will come on line in the next few years.

Source mm2: the molecular spectrum of mm2 shows emission from several species, with moderately excited $\left(E_{\text {up }} \leq\right.$ $130 \mathrm{~K})$ methanol lines. Linewidths are comparable to the one of the components in $\mathrm{mm} 1$, and emission is at $v=-15.3 \mathrm{~km} \mathrm{~s}^{-1}$. However, stronger emission is detected in several methanol lines at $\left(1.2^{\prime \prime}, 0.6^{\prime \prime}\right)$ off of $\mathrm{mm} 2\left(\alpha_{2000}=05^{\mathrm{h}} 39^{\mathrm{m}} 12^{\mathrm{s}} .86, \delta_{2000}=\right.$ $+35^{\circ} 45^{\prime} 51^{\prime \prime} .9$ ), at a position where no continuum emission is detected (see Fig. 4). At the same position, the $\mathrm{CH}_{3} \mathrm{CN} k=$ $0,1,3$ lines are also detected, while the $7 \rightarrow 6 \mathrm{C}^{34} \mathrm{~S}$ and $10_{1,10} \rightarrow 9_{1,9} \mathrm{H}_{2} \mathrm{CS}$ transitions have a secondary emission peak in the vicinity of this position. Linewidths are of the order of $3.7 \mathrm{~km} \mathrm{~s}^{-1}$, at the same velocity of $\mathrm{mm} 2$.

To rule out the hypothesis that the different distribution of the gas with respect to the dust is not real, but due to optical depth effects, the intensity of the lines should be mapped in the wings, where the optical depth is lower. Unfortunately, the signal to noise in the $\mathrm{CH}_{3} \mathrm{CN}$ spectrum and in the SMA data, which are the two datasets less affected by line-overlapping problems, is not good enough to perform this check. However, given the range of energies of the lines showing a second peak between $\mathrm{mm} 1$ and $\mathrm{mm} 2$ (see Figs. 3 and 4), we believe that this spatial distribution is real. In Fig. 8, the $\mathrm{CH}_{3} \mathrm{CN}$ and $\mathrm{CH}_{3} \mathrm{OH}$ spectra are shown towards the offset position. In the rest of the paper, we will refer to this position as to mm2-line. The molecular spectrum of mm2 looks very similar to the one of mm2-line, but line intensities are usually a factor of 1.2 weaker than on mm2-line. Since the two positions have a separation of roughly half the beam size of our observations, we cannot rule out that part of the emission at $\mathrm{mm} 2$ is coming from $\mathrm{mm} 2$-line.

A possible interpretation for the molecular emission at mm2-line is that it is caused by sputtering or thermal evaporation of the grain mantles, followed by gas-phase reactions, due to the interaction with one or more of the outflows in the region (see Fig. 9). Although mm2-line is characterised by narrow features $\left(\Delta v_{\mathrm{LSR}} \sim 3.2 \mathrm{~km} \mathrm{~s}^{-1}\right)$, the emission is red-shifted respect to $\mathrm{mm} 1$, thus strengthening the outflow scenario. However, the detection of a high-density tracing molecule like $\mathrm{CN}_{3} \mathrm{CN}$ at this position does not easily fit with this hypothesis. Alternatively, a low-mass dense core could be responsible for this emission. In this case, an upper limit to the mass of mm2-line can be computed from the continuum emission at $1.3 \mathrm{~mm}$, assuming a temperature of $60 \mathrm{~K}$ as derived from the analysis of its methanol spectrum (see Sect. 4.3). This results in a mass of $M_{\mathrm{H}_{2}} \sim 0.08 M_{\odot}$. In Sect. 4.3, we derive a lower limit of $10^{-7}$ for the abundance of methanol at mm2-line, which is comparable to the values found in low-mass star hot cores (Maret et al. 2005). These cores are however usually warmer than $60 \mathrm{~K}$. Otherise, the enhancement of the $\mathrm{CH}_{3} \mathrm{OH}$ abundance can also be explained within the hypothesis that the emission is caused by the interaction of molecular outflows with the ambient molecular cloud.

Source mm3: the core $\mathrm{mm} 3$ presents the simplest molecular spectrum among the several dust condensations in the region. The emission associated with $\mathrm{mm} 3$ is extended and not coming from a compact core, as all the lines detected are seen as negative features, an artifact due to missing spacings. Therefore, only a qualitative analysis of these data is possible. Beuther et al. (2007a) computed its spectral energy distribution and derived an upper limit to the dust temperature of $\sim 20 \mathrm{~K}$, a value that is constrained by the weakness of the continuum emission at $690 \mathrm{GHz}$. In paragraph Sect. 4.3, we discuss the molecular spectrum at this position, and conclude that the gas around $\mathrm{mm} 3$ is cold $(<30-40 \mathrm{~K})$, and dense $\left(10^{5}-10^{6} \mathrm{~cm}^{-3}\right)$. Therefore, our results agree with the conclusion of Beuther et al. (2007a) that $\mathrm{mm} 3$ is a candidate of a cold massive core in an early evolutionary stage. Our observations of $\mathrm{CH}_{3} \mathrm{OH}$ and $\mathrm{C}^{34} \mathrm{~S}$ suggest that these molecules have an extended, flat distribution, and show no trace of the structure seen in the dust continuum emission, a result reminiscent of observations of low-mass starless cores (e.g., Caselli et al. 1999). Even $\mathrm{H}^{13} \mathrm{CO}^{+}$, a good tracer of quiescent gas, shows no peak on mm3 (Beuther et al. 2002a). These results suggest that $\mathrm{mm} 3$ is indeed a candidate massive starless core. However, high angular resolution observations that trace the dense gas and investigate potential signs of star formation activity are needed to test this hypothesis. 


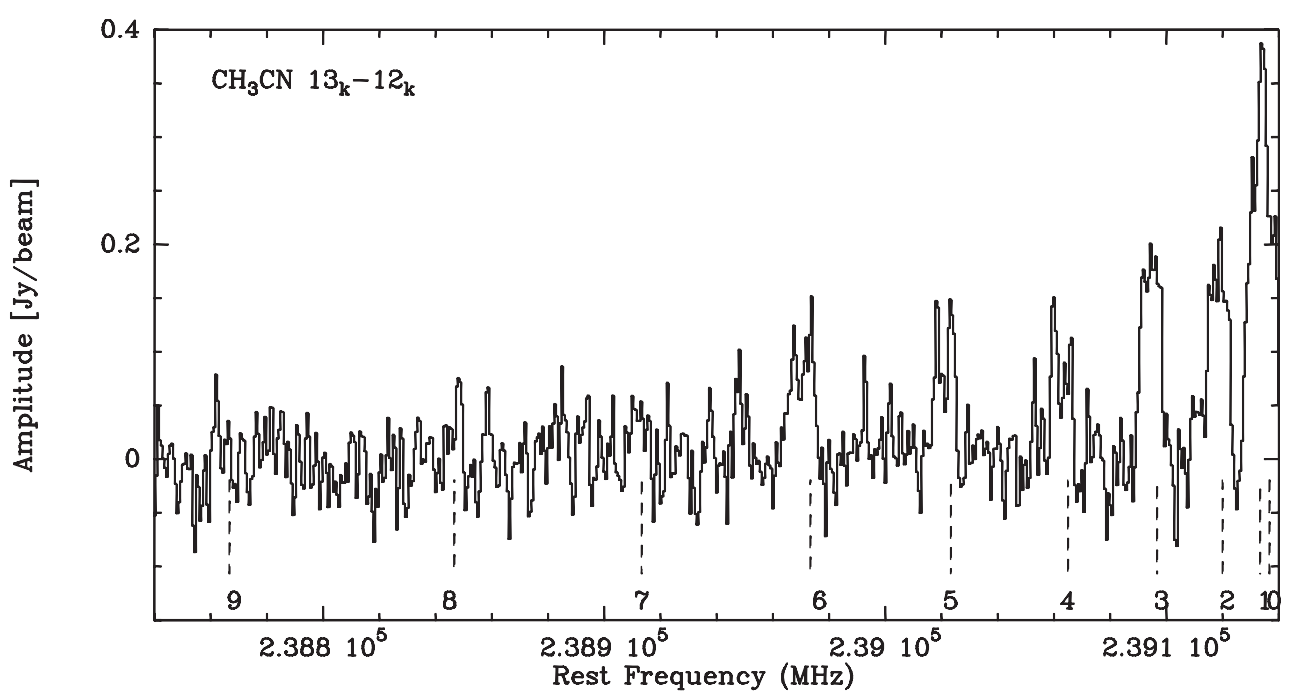

(a)

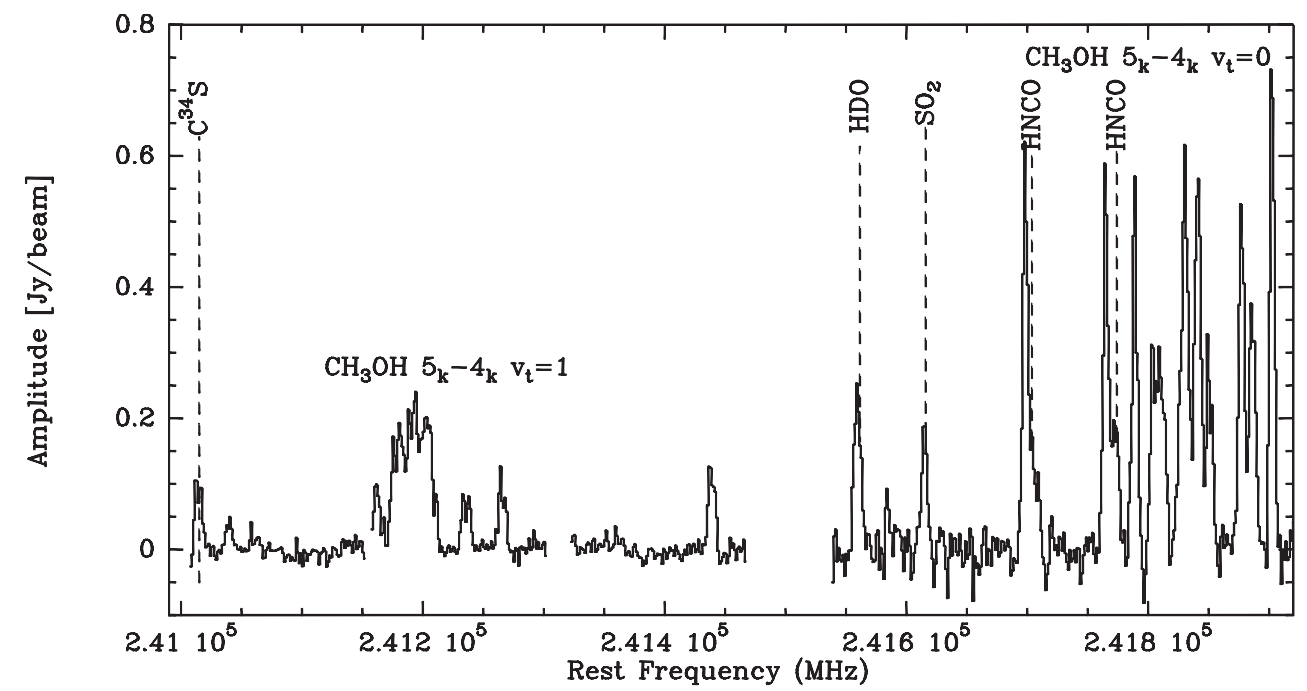

(b)

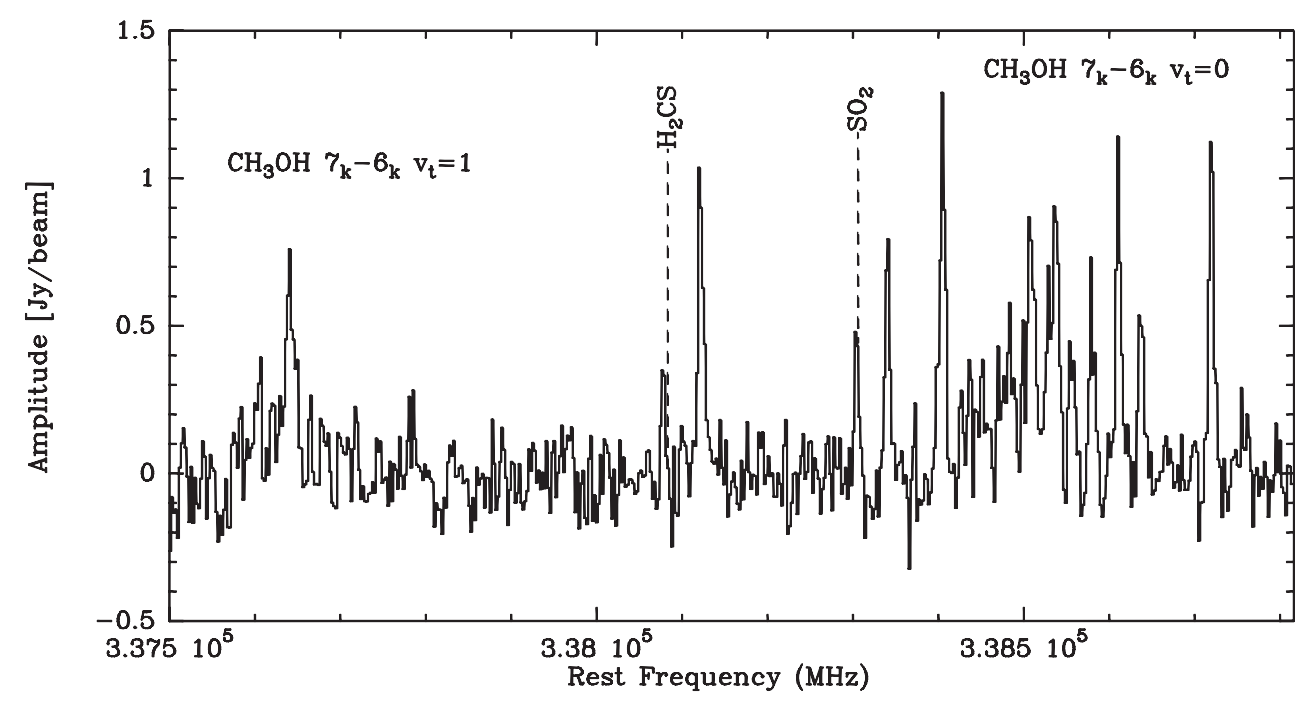

(c)

Fig. 5. Molecular spectrum of mml. The two cores, mmla and mm1b, are resolved only for $5_{k} \rightarrow 4_{k} v_{t}=1 \mathrm{CH}_{3} \mathrm{OH}$ lines at $\sim 241.2 \mathrm{GHz}$ in Fig. $5 \mathrm{~b}$. For $\mathrm{CH}_{3} \mathrm{CN}$, different $K$ numbers are marked with dashed lines in the lower part of the spectrum (Fig. 5a). The features from other molecular species are labelled with the name of the molecule. Not labelled transitions are methanol lines. 


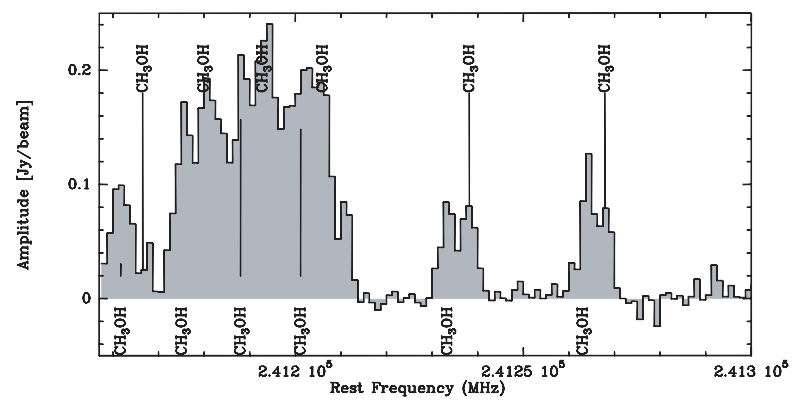

Fig. 6. Spectrum of mm1a at $241.2 \mathrm{GHz}$. The upper labels indicate the rest frequencies of the $\mathrm{CH}_{3} \mathrm{OH}$ lines for a velocity of $-17.6 \mathrm{~km} \mathrm{~s}^{-1}$, while the lower labels show the same transitions for a velocity of $-11.6 \mathrm{~km} \mathrm{~s}^{-1}$.

\section{Derivation of physical parameters}

\section{1. $\mathrm{CH}_{3} \mathrm{CN}$}

The physical properties of the gas around $\mathrm{mm} 1$ are derived by the analysis of the $13_{k} \rightarrow 12_{k} \mathrm{CH}_{3} \mathrm{CN}$ lines. The analysis was carried out with the Xclass program (discussed in Comito et al. 2005), which uses an LTE model to produce synthetic spectra, and compares them to the observations. The molecular data are from the CDMS (Müller et al. 2001) and JPL (Pickett et al. 1998) databases. The parameters defining the synthetic spectrum are: source size, rotation temperature, column density, velocity width and velocity offset (with respect to the systemic velocity of the object). Several velocity components, which are supposed to be non-interacting (i.e. the intensities add up linearly), can be used.

Given the low signal-to-noise ratio of the spectra, it is possible to perform this analysis only on $\mathrm{mm} 1$. For this position, we assumed that all the $\mathrm{CH}_{3} \mathrm{CN}$ transitions trace the same warm, dense gas around the central object and come from the two velocity components detected in several molecular species (see paragraph Sect. 3.2), although the lower excitation lines may have contribution from a more extended component (Fig. 3). Since the line profiles consist of two peaks of the same intensity, we used the same physical conditions for the two components. The source size is degenerate with temperature in the case of completely optically thick lines, and with column density for completely optically thin lines. In our case, the $k>3$ lines are optically thin, while the $k \leq 3$ are optically thick. Therefore, the degeneracy between source size and column density can be solved. Constrains on the column density of $\mathrm{CH}_{3} \mathrm{CN}$ come also from the non detection of the $13_{k} \rightarrow 12_{k} \mathrm{CH}_{3}^{13} \mathrm{CN}$ band, which is very close in frequency to the $\mathrm{CH}_{3} \mathrm{CN}$ lines. We found that the source size is constrained to $\sim 0.2^{\prime \prime}$ for each components, in agreement with the size of $0.2-0.3^{\prime \prime}$ of the linear structure of Fig. 7a. For this value, the best fit corresponds to a temperature of $220 \mathrm{~K}$, and a column density of $\sim 4 \times 10^{16} \mathrm{~cm}^{-2}$ per velocity component. The uncertainties on these values are reported in Table 5. Assuming that the $\mathrm{CH}_{3} \mathrm{CN}$ emission comes from mm1a, and using the hydrogen column density derived by Beuther et al. (2007a) for the AB configuration data at $1.2 \mathrm{~mm}$ $\left(N_{\mathrm{H}_{2}} \sim 2.2 \times 10^{24} \mathrm{~cm}^{-2}\right)$, this results in a total abundance of $\mathrm{CH}_{3} \mathrm{CN}$ relative to $\mathrm{H}_{2}$ of $4 \times 10^{-8}$, which is typical of hot cores (e.g., Hatchell et al. 1998). However, Beuther et al. (2007a) derived the $\mathrm{H}_{2}$ column density for a temperature of $50 \mathrm{~K}$, while our estimate of $T_{K}$ is higher. The abundance increases to $2 \times 10^{-7}$, when re-computing the $\mathrm{H}_{2}$ column density of mmla at $220 \mathrm{~K}$ $\left(N_{\mathrm{H}_{2}} \sim 4.6 \times 10^{23} \mathrm{~cm}^{-2}\right)$.

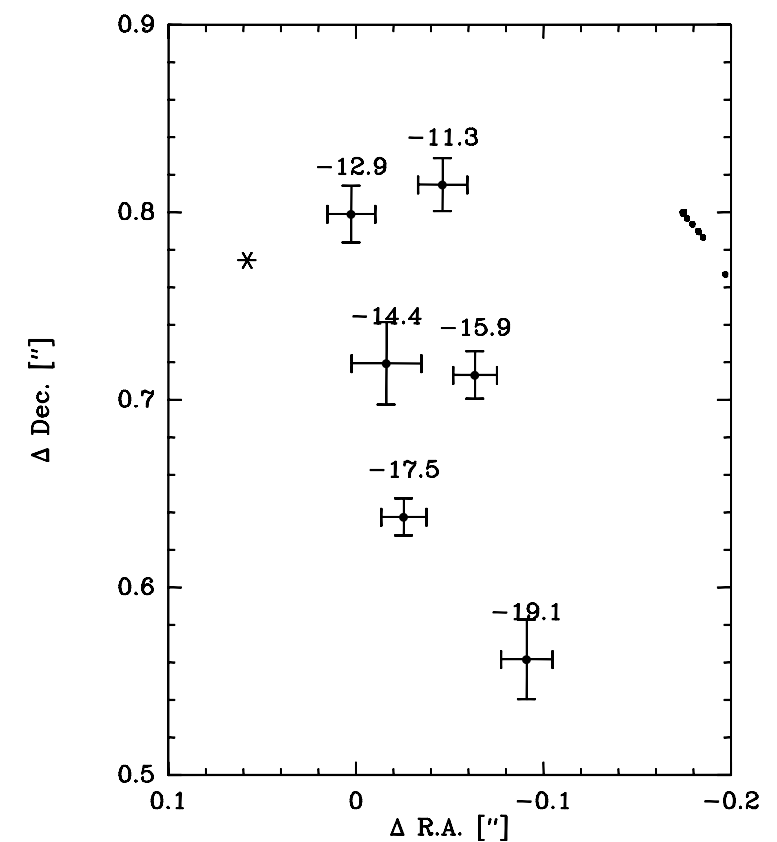

(a)

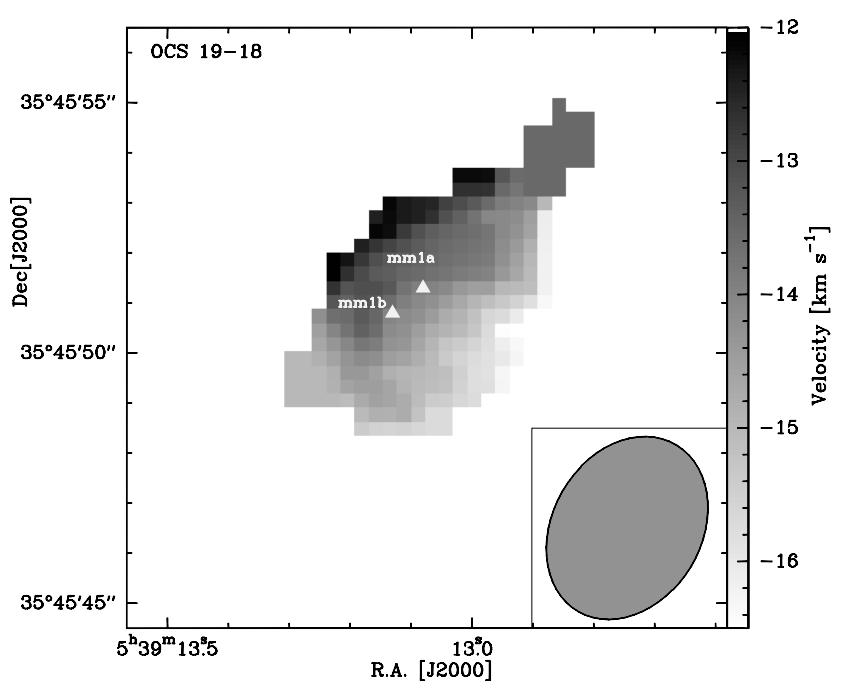

(b)

Fig. 7. a) Presented is the position-position (P-P) diagram of the $5_{-1} \rightarrow$ $4_{-1} v_{t}=1-E \mathrm{CH}_{3} \mathrm{OH}$ line. The star marks the position of the $1.3 \mathrm{~mm}$ continuum peak. The values above each mark are the LSR velocities of the corresponding channels. The error bars represent statistical errors of the fits. The error on the position of the continuum peak are negligible. The black dots on the east mark the positions of the velocity channels of the $6.7 \mathrm{GHz}$ methanol maser (Minier et al. 2000). The offset between the two linear structures can be accounted for pointing uncertainties, and error in the absolute position of the VLBI data. b) First moment map of the $19 \rightarrow 18$ OCS transition. The white triangles mark the positions of the dust condensations mmla and mmlb. The beam is shown in the bottom right corner.

\section{2. $\mathrm{SO}_{2}$}

Four $\mathrm{SO}_{2}$ transitions were detected towards the main condensation $\mathrm{mm} 1$. They range in energy of the upper level from $\sim 24$ to $200 \mathrm{~K}$; therefore, although they look point-like in all observations, they likely trace different gas around $\mathrm{mm} 1$. We analysed the $\mathrm{SO}_{2}$ emission by means of the LTE technique discussed in paragraph Sect. 4.1. Since the lines do not show any 


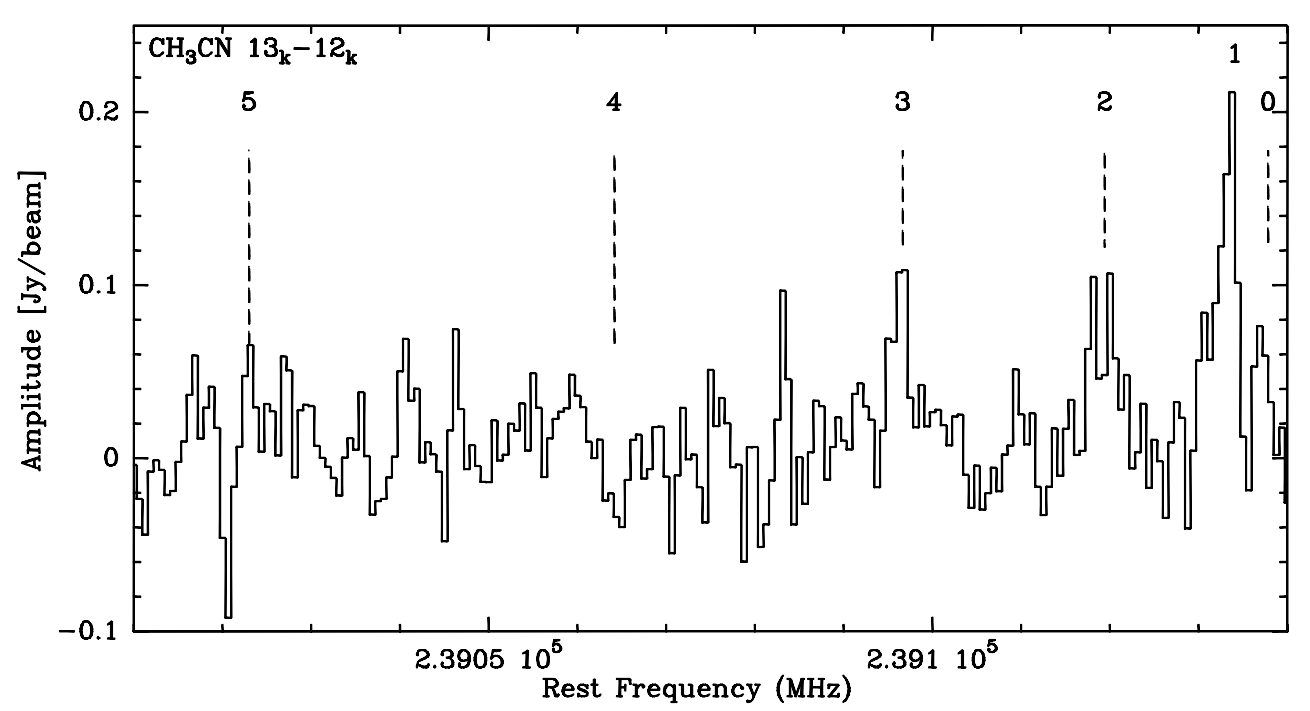

(a)

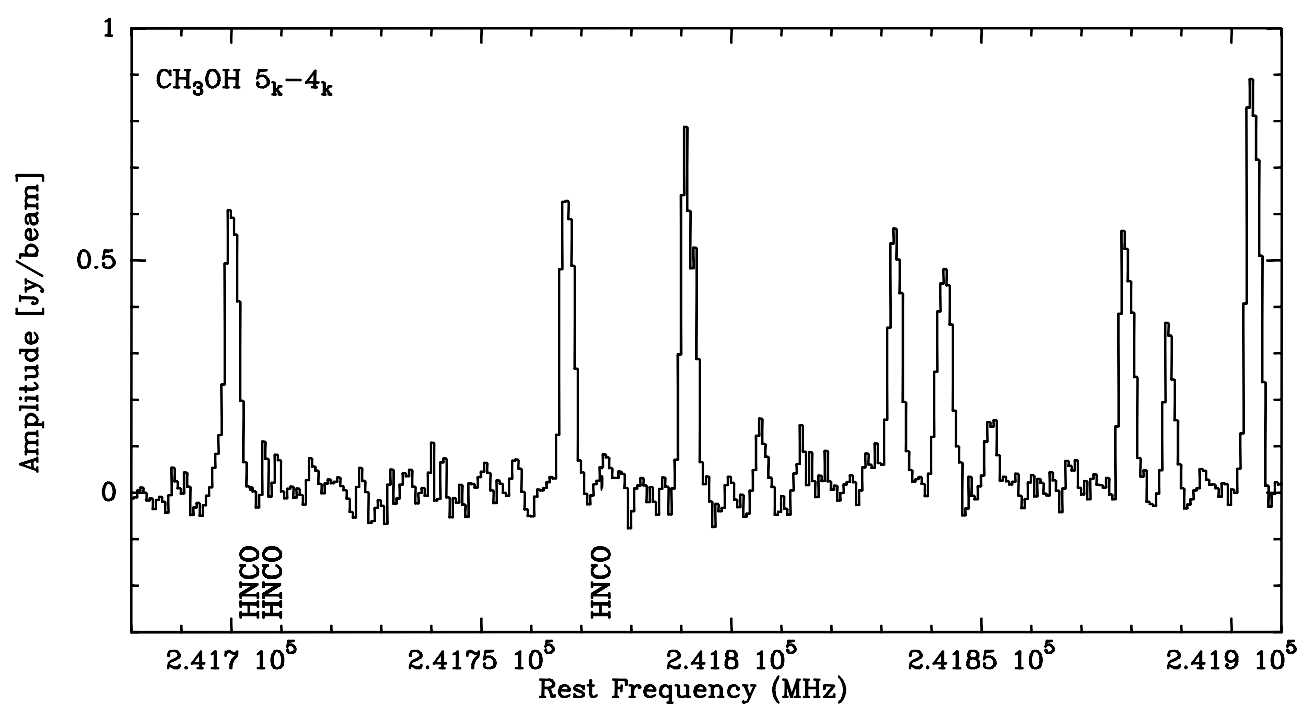

(b)

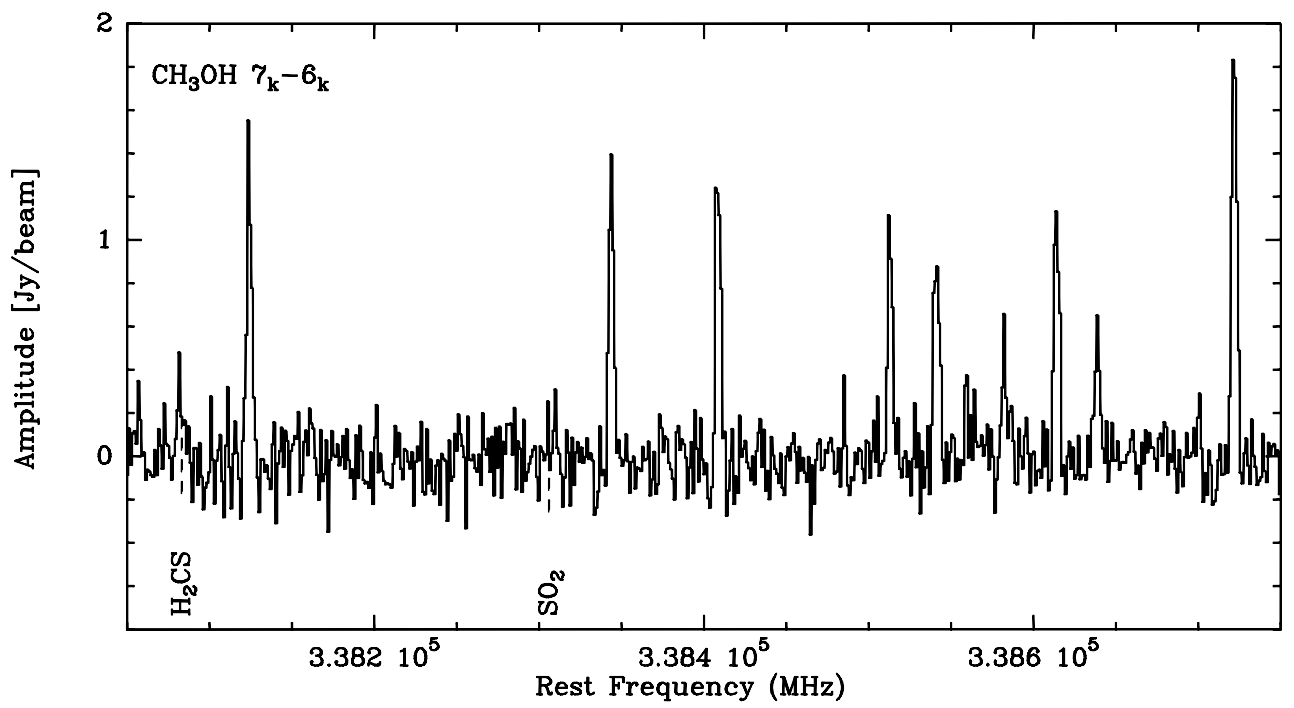

(c)

Fig. 8. Molecular spectra towards mm2-line $\left[\alpha_{2000}=05^{\mathrm{h}} 39^{\mathrm{m}} 12 \mathrm{~s} .86, \delta_{2000}=+35^{\circ} 45^{\prime} 51^{\prime \prime}\right.$. 9 ]. For $\mathrm{CH}_{3} \mathrm{CN}$, different $K$ numbers are marked with dashed lines in the upper part of the spectrum (Fig. 8a), while only the species are given for the other molecules. Not labelled transitions are methanol lines. 
Table 5. Overview of the physical parameters in IRAS 05358+3543: - indicates parameters which cannot be derived with our data.

\begin{tabular}{|c|c|c|c|c|c|c|}
\hline Core & $\begin{array}{c}\Theta \\
{\left[{ }^{\prime \prime}\right]}\end{array}$ & $\begin{array}{c}T \\
{[\mathrm{~K}]}\end{array}$ & $\begin{array}{c}n \\
{\left[\mathrm{~cm}^{-3}\right]}\end{array}$ & $\begin{array}{c}N \\
{\left[\mathrm{~cm}^{-2}\right]}\end{array}$ & $\bar{X}$ & $\begin{array}{c}N_{\mathrm{H}_{2}}^{a} \\
{\left[\mathrm{~cm}^{-2}\right]}\end{array}$ \\
\hline \multicolumn{7}{|c|}{$\mathrm{CH}_{3} \mathrm{OH}$} \\
\hline $\mathrm{mmla}^{b}$ & 0.15 & $220(75-270)$ & - & $4 \times 10^{18}\left(4 \times 10^{17}-1 \times 10^{19}\right)$ & $10^{-5}$ & $4.6 \times 10^{23}$ \\
\hline $\mathrm{mm} 2$ & 2.5 & $60(40-80)$ & $>6 \times 10^{6}$ & $4 \times 10^{15}\left(4 \times 10^{15}-5 \times 10^{15}\right)$ & $6 \times 10^{-9}$ & $2.7 \times 10^{23}$ \\
\hline mm2-line & 2.5 & $60(40-110)$ & $>6 \times 10^{6}$ & $7 \times 10^{15}\left(1 \times 10^{15}-2 \times 10^{16}\right)$ & $10^{-7}$ & $5 \times 10^{22}$ \\
\hline $\mathrm{mm} 3$ & - & $<40$ & $10^{5}-10^{6}$ & - & - & $4.9 \times 10^{24}$ \\
\hline \multicolumn{7}{|c|}{$\mathrm{CH}_{3} \mathrm{CN}$} \\
\hline $\mathrm{mm} 1$ & 1.0 & $150(>60)$ & $\mathrm{SO}_{2}$ & $1 \times 10^{16}\left(5 \times 10^{15}-5 \times 10^{16}\right)$ & $5 \times 10^{-8}$ & $2 \times 10^{23}$ \\
\hline
\end{tabular}

${ }^{a}$ Derived from the $1.3 \mathrm{~mm}$ continuum data presented by Beuther et al. (2007a) and using the temperature derived from each molecular species.

${ }^{b}$ The values refer to the total column density of the two velocity components.

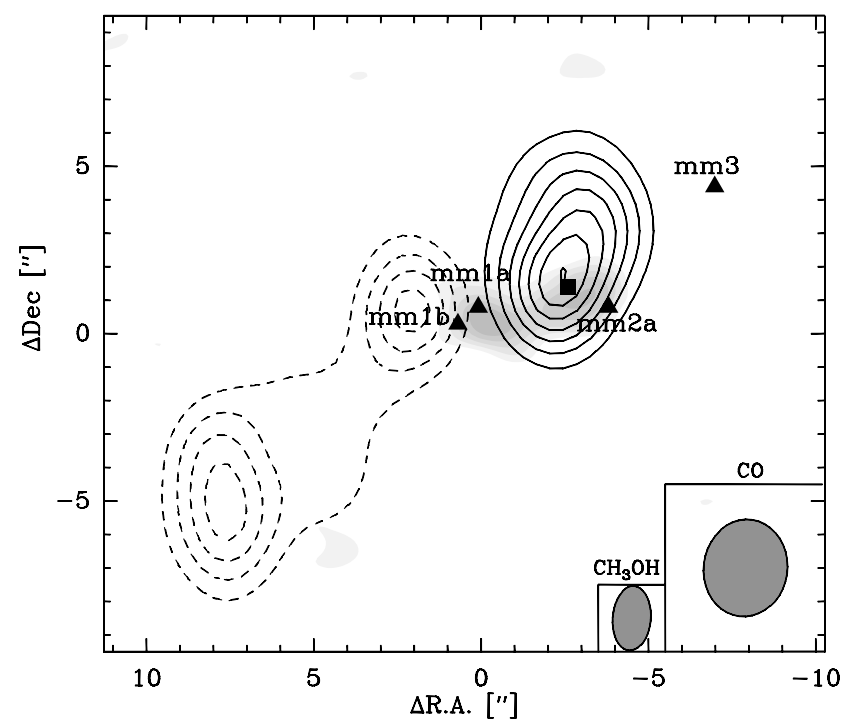

Fig. 9. In grey scale, the integrated intensity of the $7_{2} \rightarrow$ $6_{2} v_{t}=0 \mathrm{CH}_{3} \mathrm{OH}-E$ with the SMA (from $2.5 \mathrm{Jy} \mathrm{beam}^{-1} \mathrm{~km} \mathrm{~s}^{-1}$ in step of 1 ), overlaid on the $\mathrm{CO} 2 \rightarrow 1$ high velocity outflow (SMA, Beuther, priv. comm. Blue: solid lines $v=[-44,-24] \mathrm{km} \mathrm{s}^{-1}$; red: dashed lines $v=[-8,2] \mathrm{km} \mathrm{s}^{-1}$; levels from $1.5 \mathrm{Jy}^{-1} \mathrm{~km}^{-1} \mathrm{~km} \mathrm{~s}^{-1}$ in steps of 1$)$. The four triangles mark the positions of the main mm dust condensations; the square outlines the $\mathrm{mm} 2$-line position.

trace of the double-peaked profile detected in other molecular species, we used a model with one velocity component for $\mathrm{SO}_{2}$. Assuming a source size of $1^{\prime \prime}$, the best fit is found for $T=150 \mathrm{~K}$ and $N\left(\mathrm{SO}_{2}\right)=10^{16} \mathrm{~cm}^{-2}$, which corresponds to an abundance of $\mathrm{SO}_{2}$ relative to molecular hydrogen of $2 \times 10^{-8}$ using $T_{\text {dust }}=50 \mathrm{~K}\left(N_{\mathrm{H}_{2}} \sim 6.6 \times 10^{23} \mathrm{~cm}^{-2}\right)$, and of $\sim 5 \times 10^{-8}$ for a temperature of $150 \mathrm{~K}\left(N_{\mathrm{H}_{2}} \sim 2 \times 10^{23} \mathrm{~cm}^{-2}\right)$. The errors on the parameters are listed in Table 5.

\section{3. $\mathrm{CH}_{3} \mathrm{OH}$}

For the analysis of the $\mathrm{CH}_{3} \mathrm{OH}$ spectrum, we used a technique similar to the one discussed for $\mathrm{CH}_{3} \mathrm{CN}$ (Sect. 4.1) and implemented to the LVG approximation (Leurini et al. 2004, 2007). Since methanol is detected at several positions, in the following discussion the properties of each dust condensation will be discussed separately. The errors on the parameters are listed in Table 5 for each condensation.

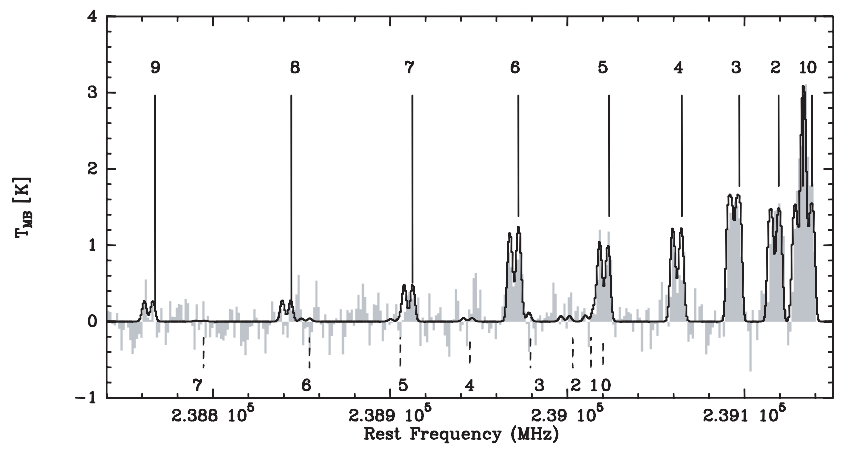

Fig. 10. Spectrum of the $13_{k} \rightarrow 12_{k} \mathrm{CH}_{3} \mathrm{CN}$ band towards the main dust condensation $\mathrm{mm} 1$; overlaid on the data, in black, the best fit synthetic spectrum. All $\mathrm{CH}_{3} \mathrm{CN}$ lines are labelled in the upper axis for the velocity component $v=-17.6 \mathrm{~km} \mathrm{~s}^{-1}$. The frequencies of the corresponding $\mathrm{CH}_{3}^{13} \mathrm{CN}$ transitions are labelled in the lower axis.

Source mmla: in our analysis of mmla, we included only emission coming from the $v_{t}=1$ lines, as their optical depth is lower than for the ground state, and their emission is confined to the gas around the dust condensation, while the $v_{t}=0$ transitions are more extended, and are affected by problems of missing flux. Moreover, the $v_{t}=1$ lines have similar level energies, while the ground state transitions in our data range from a few $\mathrm{K}$ to $\sim 200 \mathrm{~K}$, thus not tracing the same gas. The model includes the three torsionally excited bands observed, the $2_{k} \rightarrow 1_{k}$ at $3 \mathrm{~mm}$, the $5_{k} \rightarrow 4_{k}$ at $3 \mathrm{~mm}$, and the $7_{k} \rightarrow 6_{k}$ at $0.8 \mathrm{~mm}$. Infrared pumping by thermal heating of the dust is included in the form of an external grey-body (Leurini et al. 2007), with $\beta=1.6$ and $\tau_{100 \mu \mathrm{m}}=1.5$. This is necessary to explain the excitation of the torsionally excited lines, as, with very high critical densities $\left(10^{10}-10^{11} \mathrm{~cm}^{-3}\right)$ and high level energies $(T \geq 200 \mathrm{~K})$, they can be hardly populated by collisions, but trace the IR field instead. On the other hand, the ground state lines can be populated by collisions and by the infrared pumping. Leurini et al. (2007) investigated the effects of infrared pumping on the excitation of methanol, and found that, as in the case of other molecules (e.g. CS, Carroll \& Goldsmith 1981), the infrared pumping mimics the excitation by collisions of the $v_{t}=0$ lines. This results in a degeneracy between the density and the infrared field of the thermal dust which makes any determination of the density through the analysis of the ground state lines of methanol impossible, for sources like hot cores. As in the discussion in paragraph Sect. 4.1, we used two velocity components to model the data.

The best fit is reached for values very similar to the ones derived with $\mathrm{CH}_{3} \mathrm{CN}$, source sizes of $0.15^{\prime \prime}$ and a temperature of 
$220 \mathrm{~K}$. As the line intensities are very similar in the two velocity components in almost all lines, the same parameters are derived for both of them. The column density for methanol is equal to $\sim 2 \times 10^{18} \mathrm{~cm}^{-2}$ per component, which, using the $\mathrm{H}_{2}$ column density derived by Beuther et al. (2007a) for mm1 a for $50 \mathrm{~K}$, corresponds to a total abundance of methanol relative to molecular hydrogen of $2 \times 10^{-6}$, or to $10^{-5}$ if we adopt $220 \mathrm{~K}$ as dust temperature. Both values are typical of massive hot cores (Menten et al. 1986, 1988).

The fit well reproduces the intensities of the $J=5,7$ series, but it fails to correctly predict the strength of the lines at $96 \mathrm{GHz}$, which are weaker in the model than in the observations. A plausible explanation is that $3 \mathrm{~mm}$ lines come from a more extended region than the others, and therefore the real beam dilution is less than that obtained in the model with a source size of $0.15^{\prime \prime}$. With the resolution of our data, we cannot verify this hypothesis. The $2_{k} \rightarrow 1_{k}$ lines were observed in both the BCD and the AB configurations of the array, and no flux is lost in the data with the higher resolution. However, the CLEAN beam of the AB data at $3 \mathrm{~mm}$ is significantly larger than the one at $1.3 \mathrm{~mm}$, and different emission sizes between the $3 \mathrm{~mm}$ and the $1.3 \mathrm{~mm}$ lines cannot be ruled out. Moreover, the $96 \mathrm{GHz}$ lines peak at $300 \mathrm{~K}$ in energy of the upper level, while the other bands are at higher energies $(330-530 \mathrm{~K})$, and they are therefore more likely to trace the gas closer to the central heating source.

Source $m m 2$ : since no torsionally excited lines are detected toward $\mathrm{mm} 2$, the analysis on the methanol spectrum was performed on the ground state lines, and no external radiation field was used in the calculations. As discussed for mm1a, the analysis of these lines is not trivial, as they range in energy of the upper level from $35 \mathrm{~K}$ to more than $100 \mathrm{~K}$ (see Table 3), and therefore trace different regions of gas. Moreover, some $v_{t}=0$ lines have extended emission and are, thus, affected by missing fluxes problems. The analysis of the datacubes at 241.7 and $338 \mathrm{GHz}$ shows that several methanol lines have a spatial distribution similar to that of the $5_{0} \rightarrow 4_{0} v_{t}=0 \mathrm{CH}_{3} \mathrm{OH}-A$ transition (Fig. 1b). We excluded these lines from our study of $\mathrm{mm} 2$, and limited the analysis to the transitions that are peaked on the main dust cores. An asterisk in Table 3 marks the lines with extended emission, excluded from the analysis of $\mathrm{mm} 2$.

The continuum data (Beuther et al. 2007a) reveal a complex area around $\mathrm{mm} 2$, with several sub-sources which may not be of protostellar nature, but which could be caused by the outflows in the region. Given the relatively low resolution of the observations of the methanol ground state lines, the molecular emission detected toward $\mathrm{mm} 2$ could arise not only from the gas associated with the dust core $\mathrm{mm} 2 \mathrm{a}$, but also from the other sub-sources around it. Therefore, in modelling the molecular spectrum of $\mathrm{mm} 2$, we used the beam size as source size, and derived the average physical parameters of the gas over the beam. This approach is justified by the low optical depth of the methanol lines for the physical conditions found in the analysis. The same technique was used to model the methanol emission at the position $\left(\alpha_{2000}=05^{\mathrm{h}} 39^{\mathrm{m}} 12^{\mathrm{s}} .86, \delta_{2000}=+35^{\circ} 45^{\prime} 51^{\prime \prime} .9\right)$, northwest of $\mathrm{mm} 2$. Since no continuum emission is detected there, the nature of the molecular emission is unknown. The spectra toward the two positions, $\mathrm{mm} 2$ and $\mathrm{mm} 2$-line, are very similar: emission comes from the same transitions, which have similar linewidths, and emit at similar velocities. However, the emission from $\mathrm{mm} 2$-line is stronger than on $\mathrm{mm} 2$ in all methanol lines, hence suggesting that the methanol abundance is enhanced at this position. The best fit is found for a temperature of $60 \mathrm{~K}$, and a column density of $4 \times 10^{5} \mathrm{~cm}^{-2}$ and $7 \times 10^{5} \mathrm{~cm}^{-2}$ for $\mathrm{mm} 2$ and $\mathrm{mm} 2$-line, respectively. The column densities at the two positions are $4 \times 10^{15} \mathrm{~cm}^{-2}$ for $\mathrm{mm} 2$, and $7 \times 10^{15} \mathrm{~cm}^{-2}$ for $\mathrm{mm} 2$-line. The corresponding abundances of methanol relative to $\mathrm{H}_{2}$ can be calculated by using the $\mathrm{H}_{2}$ column density derived by Beuther et al. (2007a) from the BCD configuration data. The methanol abundance of $\mathrm{mm} 2$ corresponds to $6 \times 10^{-9}$, a value closer to the ones derived towards the inner regions of low-mass protostars (e.g., Maret et al. 2005), than to values found in highmass protostars (Menten et al. 1986, 1988). In Sect. 3.2, we noticed that, given the separation between $\mathrm{mm} 2$ and $\mathrm{mm} 2$-line, at least part of the emission of $\mathrm{mm} 2$ could come from the offset position. Therefore, the $\mathrm{CH}_{3} \mathrm{OH}$ column density derived for $\mathrm{mm} 2$ should be regarded as an upper limit to its true value.

An upper limit to $\mathrm{H}_{2}$ column density of $\mathrm{mm} 2$-line can be computed from the continuum emission at $1.3 \mathrm{~mm}, N \sim 5 \times$ $10^{22} \mathrm{~cm}^{-2}$ at $60 \mathrm{~K}$. This results in an abundance of $1 \times 10^{-7}$ for methanol, which is a factor of 17 higher than on $\mathrm{mm} 2$. However, a different dust temperature would result in different values for $N_{\mathrm{H}_{2}}$ and $X_{\mathrm{CH}_{3} \mathrm{OH}}$. The enhancement of methanol is usually ascribed to evaporation of icy grain mantles in the vicinity of warm embedded sources, e.g. hot cores, or to desorption of grain mantles in shocks associated to molecular outflows (e.g., Bachiller et al. 1995). Since no embedded sources are detected in the continuum emission at this position down to an upper limit of $0.08 M_{\odot}$, it seems likely that the molecular emission associated with $\mathrm{mm} 2$-line is related to the outflow activity of the region.

In the case of $\mathrm{mm} 2$, and $\mathrm{mm} 2$-line, the temperatures derived from methanol are comparable to those adopted by Beuther et al. (2007a) for the analysis of the continuum emission; therefore, only one value for the methanol abundance is given at this position.

Source mm3: as listed in Table 3, only molecular transitions from the ground state, and with upper energies of less than $70 \mathrm{~K}$ are detected toward $\mathrm{mm} 3$. Since all the lines detected toward this core are seen in absorption due to missing fluxes, with emission only in the $5_{0} \rightarrow 4_{0}-A$ and $5_{-1} \rightarrow 4_{-1}-E$ transitions on top of the negative features, only a qualitative analysis of the data is possible. We ran LVG models for several temperatures and densities, to find the range of parameters compatible with our observations. The detection of the $5_{ \pm 2} \rightarrow 4_{ \pm 2}-E$ lines, and the non detection of the higher $k$ transitions constrain the $\mathrm{H}_{2}$ density to the range $\sim 10^{5}-10^{6} \mathrm{~cm}^{-3}$, while the tentative detection of the $k=0-A$ and $k=-1-E$ lines in the $7_{k} \rightarrow 6_{k}$ band, but not of the other transitions, sets an upper limit to the temperature of $30-40 \mathrm{~K}$.

\subsection{Uncertainties on the physical parameters of the gas}

Error ranges for the various parameters can be estimated by a $\chi^{2}$ analysis, although this can only give errors within the assumptions of the model. It cannot assess errors due to the LTE and LVG assumptions and to the treatment of the gas as consisting of a finite number of non-interacting components of homogeneous conditions. As for the LTE assumption, densities in hot cores usually are in a range which makes it reasonable.

The analysis of $\mathrm{CH}_{3} \mathrm{CN}$ and $\mathrm{CH}_{3} \mathrm{OH}$ infers a source size of $\sim 0.2^{\prime \prime}$ for the gas responsible of the emission of the two velocity components found toward mmla. This value is relatively well constrained by the $\mathrm{CH}_{3} \mathrm{CN}$ band, which shows optically thick and thin lines, and by the non detection of $\mathrm{CH}_{3}^{13} \mathrm{CN}$. Since this result is in agreement with the size of the linear structure found from the fit of the positions of the velocity channels of methanol (see Fig. 7a), we fixed the source size to this value, and derived confidence levels for the estimates of the temperatures and column densities. However, one should remember that for optical thin lines, like the $v_{t}=1 \mathrm{CH}_{3} \mathrm{OH}$ transitions, the source 
size and column density are degenerate parameters, and the estimates of the column densities would change, if the source size were different than our assumption. For $\mathrm{CH}_{3} \mathrm{OH}$ toward mmla, since these data were taken with a beam of $\sim 0.7^{\prime \prime}$ and we assumed a size of $0.15^{\prime \prime}$ in the calculations, the $\mathrm{CH}_{3} \mathrm{OH}$ column density can only be a factor of 10 smaller than indicated by our results. For a source size of $0.15^{\prime \prime}$, good fits to the data are found also for lower temperatures, but higher column densities. For $T \sim 100 \mathrm{~K}$ the model requires column densities of $\geq 10^{19} \mathrm{~cm}^{-2}$, which would correspond to very high abundances relative to $\mathrm{H}_{2}$ for such temperatures $\left(X_{\mathrm{CH}_{3} \mathrm{OH}} \geq 10^{-5}\right)$. For temperatures higher than $150 \mathrm{~K}$, the methanol column density is confined to the interval $4 \times 10^{17}-2 \times 10^{18} \mathrm{~cm}^{-2}$. A lower limit to the kinetic temperature is found at $\sim 75 \mathrm{~K}$, where the torsionally excited lines of methanol are not anymore efficiently pumped.

Similarly, the column density of $\mathrm{SO}_{2}$ on $\mathrm{mm} 1$, and of $\mathrm{CH}_{3} \mathrm{OH}$ on $\mathrm{mm} 2$ and $\mathrm{mm} 2$-line would increase if they were constrained to a region smaller in size than the value used in the calculation. For example, the column density of $\mathrm{SO}_{2}$ would increase of one order of magnitude by using a sorce size of $0.2^{\prime \prime}$ as derived from the analysis of $\mathrm{CH}_{3} \mathrm{CN}$ and $\mathrm{CH}_{3} \mathrm{OH}$.

The $3 \sigma$ confidence levels derived for each parameter from the $\chi^{2}$ analysis are given in Table 5, together with their best fit values.

\section{Various stages of star formation}

The analysis of the spectral line emission in IRAS 05358 reveals four dust condensations with a maximum projected distance of $18000 \mathrm{AU}$. At least three of them show signs of active star formation. The core mmla appears to be the most evolved source of the region, associated with a hypercompact HII region, and class II methanol masers. Beuther et al. (2007a) derived the Lyman continuum flux of mmla corresponding to a B1 ZAMS star of $13 M_{\odot}$, and a large reservoir of accreting material $\left(\sim 10 M_{\odot}\right)$. However, this estimate is obtained from relatively low resolution data, where the cores mmla and mmlb are not spatially resolved. From high resolution data, which are however affected by missing fluxes, the estimate to the mass of mmla is $1 M_{\odot}$, and $0.6 M_{\odot}$ for mm1b. Our observations suggest that mmla also harbours a candidate circumstellar disk. A rough estimate of the luminosity of mmla can be derived through the Stefan-Boltzmann formula, assuming a radius of $180 \mathrm{AU}$, and a temperature of $220 \mathrm{~K}$. By adding the values for the two components, the total luminosity of mmla is $L \sim 6000 L_{\odot}$, in agreement with the luminosity of $10^{3.72} L_{\odot}$ of a B1 ZAMS star (Panagia 1973). Since the total luminosity of the cluster is $6300 L_{\odot}$, this result supports the interpretation that $\mathrm{mm} 1 \mathrm{a}$ is the main powerhouse of the region.

Viti et al. (2004) derived the abundances of several molecules in the gas phase, as function of the age, and of the mass of the heating central object. The abundances of $\mathrm{CH}_{3} \mathrm{OH}, \mathrm{CH}_{3} \mathrm{CN}$, and $\mathrm{SO}_{2}$ of mmla are in agreement with a $15 M_{\odot}$ star of approximately $10^{4.6} \mathrm{yr}$, although the best fit value for the column density of $\mathrm{CH}_{3} \mathrm{OH}$ is higher than the theoretical values, but still consistent with the models within the uncertainties of the fit. Decreasing the mass of the heating source in the models results in increasing its age. The value of $10^{4.6} \mathrm{yr}$ is in very good agreement with the value obtained by Beuther et al. (2002a) for the dynamical timescale of the molecular outflows originating from the vicinity of $\mathrm{mm} 1(t \sim 37000 \mathrm{yr})$.

The physical parameters of the gas derived from our analysis, (temperature and abundances) are similar to hot cores, thus implying that mmla has already reached this evolutionary stage.
However, its molecular spectrum differs from the one of typical massive hot cores. Beuther et al. (2007b) studied the hot core in G29.96-0.02 ( $L \sim 9 \times 10^{4} L_{\odot}$, Olmi et al. 2003) with the SMA, with the same frequency setup we used for our SMA observations. Although the linear resolution reached for G29.96 is comparable to ours, the spectrum of G29.96 shows stronger methanol lines than IRAS $05358+3543$ and higher excitation transitions; moreover, molecular species which are detected in G29.96 are not in our dataset. The same is found when comparing our observations with Orion-KL $\left(L \sim 10^{5} L_{\odot}\right)$, even with the poor angular resolution of the CSO telescope (Schilke et al. 1997). Possible explanations for these differences are that mmla is indeed a) in an earlier evolutionary stage than the other two sources, at the beginning of the hot core phase, and will eventually reach their chemical richness; or $b$ ) hosts a less luminous hot core, which will never produce the rich chemistry of G29.96-0.02 or Orion-KL. Both interpretations render mmla an interesting source, since they collocate it in a different region of the ageluminosity plane of hot cores, which still has not received much attention. Studies of large samples of hot cores at the same linear resolution, and with the same frequency setups (similar to that of Beuther 2007, but on a larger sample of sources) are necessary to better understand this evolutionary phase of star formation. Particularly interesting is the comparison with observations of hot cores in the vicinity of low- and intermediate-mass protostars, which could clarify whether the poorness of the chemistry in IRAS $05358+3543$ is due to its age or it is rather an intrinsic property of less luminous hot cores.

Although the mm-wavelength thermal continuum emission of mm $1 \mathrm{~b}$ is similar in intensity and extent to that of mmla, even if not as strong, mm1b is not associated with any cm continuum emission down to a threshold of $1 \mathrm{mJy}$, while mmla is. Moreover, the molecular spectrum of $\mathrm{mm} 1 \mathrm{~b}$ seems very different from that of mm1a. Of all the species we observed, only $\mathrm{C}^{34} \mathrm{~S}$ is clearly found in $\mathrm{mm} 1 \mathrm{~b}$. This is not a bias of our observation as other observations confirm our results. Class II methanol masers and a mid-IR source are detected only towards mmla, hence confirming the different nature of the two cores. The core mmlb could be at an earlier stage of evolution, when the heating from the central star still has not produced any complex chemistry, nor formed a hypercompact HII region.

The region around $\mathrm{mm} 2$ represents a challenge for the interpretation of the continuum emission (Beuther et al. 2007a), as well as for the spectral data. Several sub-sources are identified around the main dust condensation, which are probably caused by the interaction of molecular outflows with the molecular cloud. Their total mass corresponds to $5 M_{\odot}$. The molecular emission associated with the dust core does not show any sign of highly excited lines, but points out at a warm $(T \sim 60 \mathrm{~K})$, dense $\left(n>6 \times 10^{6} \mathrm{~cm}^{-3}\right)$ gas with a methanol abundance of $\sim 7 \times 10^{-9}$. The $X_{\mathrm{CH}_{3} \mathrm{OH}}$ of mm2 is more similar to the values derived in star forming regions of lower mass than to high-mass young stellar objects. Given the total mass of the region, and the low abundance of methanol, we speculate that $\mathrm{mm} 2$ is a low-intermediate mass protostar. However, the spatial resolution of our data does not allow us to verify whether or not at least part of the emission from $\mathrm{mm} 2$ is indeed not coming from mm2-line.

The coldest and least active core of the region is the dust condensation $\mathrm{mm} 3$. Its $\mathrm{mm}$ continuum emission shows that $\mathrm{mm} 3$ is a compact, unresolved source even at the highest resolution reached by our observations (Beuther et al. 2007a). No compact molecular emission is detected from this core, but all the lines observed toward it are seen as negative features due to the filtering out of large structures of the data. The analysis of the 
spectral energy distribution (Beuther et al. 2007a), and of the gas around the core confirm that $\mathrm{mm} 3$ is a dense $\left(n>10^{5} \mathrm{~cm}^{-3}\right)$, cold $(T<40 \mathrm{~K})$ condensation, surrounded by a large reservoir of accreting material $\left(M \sim 19 M_{\odot}\right.$, Beuther et al. 2007a). The extended, flat distribution of molecules like $\mathrm{CH}_{3} \mathrm{OH}, \mathrm{C}^{34} \mathrm{~S}$ and $\mathrm{H}^{13} \mathrm{CO}^{+}$, with no trace of the structure seen in the dust continuum emission, is reminiscent of low-mass starless cores, where C-bearing molecules are freeze-out onto the grain mantles in a cold and dense environments (e.g., Caselli et al. 1999). Could mm3 be a massive starless core? Massive starless cores can be found in the vicinity of active sites of massive star-formation, and should be quiescent displaying no signposts of star formation. Its properties suggest that $\mathrm{mm} 3$ is indeed a massive cold core in a very early evolutionary stage. However, with the observations we currently have we cannot exclude that star formation have already started in this core. In particular, we cannot exclude that $\mathrm{mm} 3$ is the driving source of one of the outflows detected in the region.

\section{Summary}

Our new interferometric data resolve at least four cores in the high-mass protocluster IRAS $05358+3543$. By analysing the molecular spectrum of each condensations, we characterised the properties of the gas surrounding it. Our main results are summarised in the following:

- the main powerhouse of the region, mmla, harbours a hot core with $T \sim 220 \mathrm{~K}$, and the central heating source has a chemical timescale of $10^{4.6} \mathrm{yr}$. Our data suggest that $\mathrm{mm} 1 \mathrm{a}$ might host a massive circumstellar disk;

- although the properties of the mm continuum emission of $\mathrm{mm} 1 \mathrm{~b}$ are very similar to the one of mmla, the two sources differ significantly in the $\mathrm{cm}$ and mid-infrared spectrum. This core could be in an earlier stage of star formation than mm1a, since no molecular emission is detected toward it, with the only exception of the $5 \rightarrow 4 \mathrm{C}^{34} \mathrm{~S}$ transition;

- given the low abundance of methanol, $\mathrm{mm} 2$ could be a lowintermediate mass protostar;

- strong emission is detected in several molecular species to the north-west of $\mathrm{mm} 2$, at a position where no continuum emission is detected. We suggest that this is caused by the interaction of the outflows with the ambient molecular cloud;

- the least active source, $\mathrm{mm} 3$, could be a starless massive core, since it is cold $(T<20 \mathrm{~K})$, with a large reservoir of accreting material $\left(M \sim 19 M_{\odot}\right)$, but no molecular emission peaks on it.

Acknowledgements. We would like to thank Jan Martin Winters and Roberto Neri for their helpful support during the reduction of the PdBI data, and Gabriel Paubert for performing the IRAM $30 \mathrm{~m}$ observations in service mode. We thank Vincent Minier for providing us with the positions of the velocity channels of the $6.7 \mathrm{GHz}$ methanol maser line. H.B. acknowledges financial support by the Emmy-Noether-Program of the Deutsche Forschungsgemeinschaft (DFG, grant BE2578).

\section{References}

Bachiller, R., Liechti, S., Walmsley, C. M., \& Colomer, F. 1995, A\&A, 295, L51 Beckwith, S. V. W., \& Sargent, A. I. 1993, ApJ, 402, 280

Beuther, H. 2007, in IAU Symp., 237, 148

Beuther, H., Schilke, P., Gueth, F., et al. 2002a, A\&A, 387, 931
Beuther, H., Schilke, P., Menten, K. M., et al. 2002b, ApJ, 566, 945 Beuther, H., Schilke, P., Sridharan, T. K., et al. 2002c, A\&A, 383, 892 Beuther, H., Walsh, A., Schilke, P., et al. 2002d, A\&A, 390, 289 Beuther, H., Zhang, Q., Sridharan, T. K., \& Chen, Y. 2005, ApJ, 628, 800 Beuther, H., Leurini, S., Schilke, P., et al. 2007a, A\&A, 466, 1065 Beuther, H., Zhang, Q., Bergin, E. A., et al. 2007b, A\&A, 468, 1045 Carroll, T. J., \& Goldsmith, P. F. 1981, ApJ, 245, 891

Caselli, P., Walmsley, C. M., Tafalla, M., Dore, L., \& Myers, P. C. 1999, ApJ, 523, L165

Cesaroni, R., Felli, M., Testi, L., Walmsley, C. M., \& Olmi, L. 1997, A\&A, 325, 725

Cesaroni, R., Felli, M., Jenness, T., et al. 1999, A\&A, 345, 949

Cesaroni, R., Neri, R., Olmi, L., et al. 2005, A\&A, 434, 1039

Cesaroni, R., Galli, D., Lodato, G., Walmsley, C. M., \& Zhang, Q. 2007, in Protostars and Planets V, ed. B. Reipurth, D. Jewitt, \& K. Keil, 197

Chen, H.-R., Welch, W. J., Wilner, D. J., \& Sutton, E. C. 2006, ApJ, 639, 975

Comito, C., Schilke, P., Phillips, T. G., et al. 2005, ApJS, 156, 127

Faúndez, S., Bronfman, L., Garay, G., et al. 2004, A\&A, 426, 97

Fuller, G. A., Williams, S. J., \& Sridharan, T. K. 2005, A\&A, 442, 949

Hatchell, J., Thompson, M. A., Millar, T. J., \& MacDonald, G. H. 1998, A\&AS, 133,29

Hatchell, J., Fuller, G. A., Millar, T. J., Thompson, M. A., \& Macdonald, G. H. 2000, A\&A, 357, 637

Hunter, T. R., Brogan, C. L., Megeath, S. T., et al. 2006, ApJ, 649, 888

Leurini, S., Schilke, P., Menten, K. M., et al. 2004, A\&A, 422, 573

Leurini, S., Schilke, P., Wyrowski, F., \& Menten, K. M. 2007, A\&A, 466, 215

Longmore, S. N., Burton, M. G., Minier, V., \& Walsh, A. J. 2006, MNRAS, 369, 1196

Müller, H. S. P., Thorwirth, S., Roth, D. A., \& Winnewisser, G. 2001, A\&A, 370, L49

Maret, S., Ceccarelli, C., Tielens, A. G. G. M., et al. 2005, A\&A, 442, 527

Megeath, S. T., Wilson, T. L., \& Corbin, M. R. 2005, ApJ, 622, L141

Menten, K. M. 1991, ApJ, 380, L75

Menten, K. M., Walmsley, C. M., Henkel, C., \& Wilson, T. L. 1986, A\&A, 157, 318

Menten, K. M., Walmsley, C. M., Henkel, C., \& Wilson, T. L. 1988, A\&A, 198, 253

Minier, V., Booth, R. S., \& Conway, J. E. 2000, A\&A, 362, 1093

Molinari, S., Brand, J., Cesaroni, R., \& Palla, F. 1996, A\&A, 308, 573

Molinari, S., Brand, J., Cesaroni, R., Palla, F., \& Palumbo, G. G. C. 1998, A\&A, 336, 339

Molinari, S., Brand, J., Cesaroni, R., \& Palla, F. 2000, A\&A, 355, 617

Olmi, L., \& Cesaroni, R. 1999, A\&A, 352, 266

Olmi, L., Cesaroni, R., Hofner, P., et al. 2003, A\&A, 407, 225

Panagia, N. 1973, AJ, 78, 929

Pickett, H. M., Poynter, R. L., Cohen, E. A., et al. 1998, J. Quant. Spectrosc. Radiat. Transf., 60

Sault, R. J., Teuben, P. J., \& Wright, M. C. H. 1995, in Astronomical Data Analysis Software and Systems IV, ASP Conf. Ser., 77, 433

Schilke, P., Groesbeck, T. D., Blake, G. A., \& Phillips, T. G. 1997, ApJS, 108, 301

Schuster, K.-F., Boucher, C., Brunswig, W., et al. 2004, A\&A, 423, 1171

Shepherd, D. S., Yu, K. C., Bally, J., \& Testi, L. 2000, ApJ, 535, 833

Snell, R. L., Dickman, R. L., \& Huang, Y.-L. 1990, ApJ, 352, 139

Sridharan, T. K., Beuther, H., Schilke, P., Menten, K. M., \& Wyrowski, F. 2002, ApJ, 566, 931

Tofani, G., Felli, M., Taylor, G. B., \& Hunter, T. R. 1995, A\&AS, 112, 299

Viti, S., Collings, M. P., Dever, J. W., McCoustra, M. R. S., \& Williams, D. A. 2004, MNRAS, 354, 1141

Walsh, A. J., Hyland, A. R., Robinson, G., \& Burton, M. G. 1997, MNRAS, 291, 261

Walsh, A. J., Burton, M. G., Hyland, A. R., \& Robinson, G. 1998, MNRAS, 301, 640

Walsh, A. J., Burton, M. G., Hyland, A. R., \& Robinson, G. 1999, MNRAS, 309, 905

Weigelt, G., Balega, Y., Preibisch, T., et al. 1999, A\&A, 347, L15

Williams, S. J., Fuller, G. A., \& Sridharan, T. K. 2004, A\&A, 417, 115

Williams, S. J., Fuller, G. A., \& Sridharan, T. K. 2005, A\&A, 434, 257

Wyrowski, F., Schilke, P., Walmsley, C. M., \& Menten, K. M. 1999, ApJ, 514, L43

Zhang, Q., Hunter, T. R., Brand, J., et al. 2001, ApJ, 552, L167

Zhang, Q., Hunter, T. R., Brand, J., et al. 2005, ApJ, 625, 864

Zhang, Q., Hunter, T. R., Beuther, H., et al. 2007, ApJ, 658, 1152 